Penultimate draft of the paper forthcoming in the Logic Journal of the IGPL

\title{
Theories of truth based on four-valued infectious logics
}

\author{
Bruno Da Ré1,2, Federico Pailos ${ }^{1,2}$, and Damian Szmuc ${ }^{1,2}$ \\ ${ }^{1}$ Department of Philosophy, University of Buenos Aires, Argentina \\ 2 IIF-SADAF, National Scientific and Technical Research Council (CONICET), Argentina
}

\begin{abstract}
Infectious logics are systems which have a truth-value that is assigned to a compound formula whenever it is assigned to one of its components. This paper studies four-valued infectious logics as the basis of transparent theories of truth. This take is motivated (i) as a way to treat different pathological sentences (like the Liar and the Truth-Teller) differently, namely, by allowing some of them to be truth-value gluts and some others to be truthvalue gaps, and (ii) as a way to treat the semantic pathology suffered by at least some of these sentences as infectious. This leads us to consider four distinct four-valued logics: one where truth-value gaps are infectious, but gluts are not; one where truth-value gluts are infectious, but gaps are not; and two logics where both gluts and gaps are infectious, in some sense. Additionally, we focus on the proof-theory of these systems, by offering a discussion of two related topics. On the one hand, we prove some limitations regarding the possibility of providing standard Gentzen sequent calculi for these systems, by dualizing and extending some recent results for infectious logics. On the other hand, we provide sound and complete four-sided sequent calculi, arguing that the most important technical and philosophical features taken into account to usually prefer standard calculi are, indeed, enjoyed by the four-sided systems.
\end{abstract}

\section{Introduction}

Infectious logics are - intuitively speaking - logics in which there is a truth-value that is assigned to a compound formula whenever it is assigned to one of its components. In this sense, infectious logics have truth-values that behave according to the motto 'one bad apple spoils the whole barrel'. Paradigmatic examples of infectious logics are (Paracomplete) Weak Kleene Logic $\mathbf{K}_{\mathbf{3}}^{\mathbf{w}}$ and Paraconsistent Weak Kleene Logic PWK, characterized by the weak truth-tables due to Kleene in [45] (for more details on the former, see [45] and on the latter see [20]).

From a philosophical point of view, during the 1930s and 1940s it has been argued that infectious logics are useful to formalize distinctive aspects of reasoning about nonsense and mean- 
inglessness, alongside with paradoxical and vague statements e.g. in [13], [42]. ${ }^{1}$ More recently, however, it has also been argued that they are useful to formalize aboutness-preserving entailments and inferences [52], [35], [20], [72], pooling procedures for expert opinions and database reasoning [38], Buddhist philosophy [61], [63], faulty computers and programming languages [34], among many other deeply interesting philosophical issues. Additionally, from a more algebraic point of view, these systems were studied in relation with various structures such as bilattices, trilattices and operations on them [38], [36], power algebras and power matrices [44], bisemilattices [15], and plurivalent logics [62], [44], [50], [51]. ${ }^{2}$

All these topics involve philosophical or conceptual reflections that we carry out in natural language. Yet another concept we ubiquitously talk about when reasoning in natural language is truth. As [65] and [43] say, we indispensably use the truth predicate in natural language to make some assertions that we would not be otherwise able to make, like e.g. "All $P$ s are true" and "Some $P$ s are true", where the objects satisfying $P$ are taken to be infinitely many. Although it is commonly assumed (as in e.g. [43], [59], [37]) that for the truth predicate to have its intended functioning, it must be the case that it satisfies Tarski's T-Schema, recently some scholars (cf. [12], [11], [69].) have decided to take the defining transparent, 'see-through' or 'disquotational' property of truth to be the intersubstitutivity of $A$ and ' $A$ is true' in all non-opaque contexts.

Thus, an interesting question is: can we conceive some reasons to entertain infectious logics to reason with a transparent truth predicate? The aim of this paper is to give an affirmative answer to that question, by focusing specially in the case of formal theories of truth based on four-valued infectious logics. This take is motivated, first, as a way to treat different pathological sentences (like the Liar and the Truth-Teller) differently, namely, by allowing some of them to be truth-value gluts and some others to be truth-value gaps; and, secondly, as a way to treat the semantic pathology suffered by at least some of these sentences as an infectious feature. As suggested by the results in [14] that we dualize and extend, it is not easy to find proof-theoriessuch as e.g. standard two-sided sequent calculi-for these peculiar infectious systems. In the present paper we not only introduce four-sided sequent calculi for the target logics, but also provide philosophical reasons for preferring them over other possible non-standard calculi for the systems in question. Thus, this paper also enjoys the conceptual advantage of presenting sound and complete proof-theories for these four-valued infectious logics for the first time. ${ }^{3}$

It should be mentioned that some studies with theories of truth based on three-valued infectious logics have been done, i.e. on (Paracomplete) Weak Kleene Logic $\mathbf{K}_{3}^{\mathbf{w}}$ and Paraconsistent Weak Kleene Logic PWK. Analysis of the former were carried out by e.g. Martin and Woodruff [48], Gupta and Martin [41], Gupta and Belnap [40], Kripke [46] and Fitting [38] (among many others), while considerations of the latter are a bit more scarce and limited to the work of Barrio et al. [8]. In what follows we will not be focusing on three-valued systems, although given the four-valued ones are their subsystems we will cite, mention, use and even prove new results regarding them, as an auxiliary means towards our scrutiny of the theories of truth based on four-valued infectious logics.

The paper is structured as follows. In Section 2, we give some philosophical arguments for considering the adoption of theories of truth based on four-valued infectious logics. In Section 3

\footnotetext{
${ }^{1}$ Thus, as an anonymous referee points out, the role played by, respectively, Dmitri Bochvar and Sören Halldén in the development of the three-valued logics $\mathbf{K}_{3}^{\mathbf{w}}$ and $\mathbf{P W K}$ should not be overlooked - for these systems can be seen as the $\{\neg, \wedge, \vee\}$-fragments of the respective logics of nonsense due to these authors, discussed in [13] and [42].

${ }^{2}$ With these references we do not want to give the impression that we think there is a long and equal history of work on infectious logics. Much to the contrary, we would like to highlight, first, that although systems of these sort count with a prehistory, this is indeed a flourishing area; and, second, that -in the words of an anonymous referee- there is nothing wrong with working on something relatively new.

${ }^{3}$ In [8] semantic arguments were given for this positive claim, but no proof-theoretic investigations were conducted.
} 
we present a collection of infectious logics, together with the formal theories of truth based on them. In Section 4 we review and generalize some results regarding the availability of standard sequent calculi for these infectious logics, motivating the adoption of multi-sided sequent calculi. In Section 5 we present four-sided sequent calculi for each of the target systems. Finally, Section 6 considers functionally complete extensions for these logics and theories of truth based on these extensions, while Section 7 presents some concluding remarks.

\section{Why theories of truth based on four-valued infectious logics?}

\subsection{Why four-valued logics?}

Assume you have a language which has an unary truth predicate $\operatorname{Tr}$, and assume that for every sentence $A$ of the language you also have names of the form $\langle A\rangle$ for those sentences, i.e. quotation-names. This allows, for each sentence $A$ of the language, to have another sentence $\operatorname{Tr}(\langle A\rangle)$ that constitutes or represents the truth-predication on $A$. Assume, additionally, that the truth predicate $\operatorname{Tr}$ satisfies transparency, i.e. that $A$ and $\operatorname{Tr}(\langle A\rangle)$ are intersubstitutable in all non-opaque contexts. ${ }^{4}$ Now, it is certainly possible to conceive, in such a language, sentences like $\tau$ and $\lambda$.

The sentence $\tau$ is true

The sentence $\lambda$ is false

If we reason within classical logic's bounds, we arrive at two important results: on the one hand, the sentence $\tau$ cannot be non-arbitrarily ${ }^{5}$ assigned the value true or the value false; on the other hand, the sentence $\lambda$ cannot be consistently assigned the value true or the value false. In common terms, both $\tau$ and $\lambda$ are therefore taken to be pathological sentences, where the pathology referred is some kind of semantic oddness. In more appropriate terms, it is said that inconsistent sentences like $\lambda$ are paradoxical, whereas indeterminate sentences like $\tau$ are hypodoxical (see [30], [31], [32] and [66] for this terminology).

These semantic conundrums led many scholars - who believe the truth predicate should behave transparently, and who also think that self-referential sentences like the above rightfully arise in natural languages - to go beyond the limits of classical logic and to embrace some other non-classical systems to reason with the truth predicate. Of the many traditions that sprung from this non-classical attempts, we will concentrate on two: the paracomplete and the paraconsistent.

The paracomplete approach is adopted by those logicians and philosophers who thought of pathological sentences as being neither-true-nor-false or indeterminate, that is, as being truthvalue gaps. Among the most contemporary authors that adopted this idea are Bochvar [13],

\footnotetext{
${ }^{4}$ Let us recall that non-opaque contexts are, simply, contexts which are not opaque- while opaque contexts themselves are expressions where substitution of co-referential terms does not preserve the truth-value of the whole expression. For example, "Lois Lane believes $x$ is a superhero" is an opaque context, for "Lois Lane believes Superman is a superhero" might be true while "Lois Lane believes Clark Kent is a superhero" might be false, even though 'Superman' and 'Clark Kent' are co-referential expressions.

${ }^{5}$ Meaning by "non-arbitrarily" that there is no independent reason to affirm that $\tau$ is true, or that it is false, although were we to suppose that $\tau$ is true, or that it is false, we would inevitably conclude- by the transparency of the truth predicate - that $\tau$ is indeed true or, respectively, that it is indeed false. This is usually formally represented in the context of transparent theories of truth, by saying that sentences like $\tau$ can receive different truth-values at different maximal Kleene-Kripke fixed-points that characterize the extension and anti-extension of the truth predicate. For more on this see e.g. [37] and [12]. Thanks to an anonymous referee for urging us to provide clarification on this.
} 
Kripke [46], Field [37], and many others. For instance, Bochvar considered pathological sentences present in set-theoretic and semantic paradoxes to be meaningless and, thus, non-truth-apt. Meanwhile, e.g. Kripke considered pathological sentences to be ungrounded and, thus, not to be determined by any worldly facts, thereby lacking a truth-value.

The paraconsistent approach is adopted by those who think of pathological sentences as being both-true-and-false or inconsistent, that is, as being truth-value gluts. Among the most contemporary authors that held this view at some point are Asenjo [3], [4], Priest [56], [57], [60], Routley [71], Beall [12], and many others. For instance, Priest is commonly reputed as considering pathological sentences to be true contradictions - a view that not all the advocates of the paraconsistent approach subscribe to.

Formally speaking, the paracomplete and the paraconsistent traditions seem to suggest that work with e.g. transparent theories of truth is better conducted with an underlying three-valued logic counting with the classical truth-values and an additional third non-classical value. Namely, in the case of those in the paracomplete tradition, a truth-value representing a gap, and in the case of those in the paraconsistent tradition, a truth-value representing a glut. In both cases the non-classical values are fixed-points for negation ${ }^{6}$, the main difference being that while in the paracomplete case the value in question is usually taken to be undesignated, in the paraconsistent case the value in question is usually taken to be designated. To truth-values behaving in this way we might refer without loss of generality, in what follows, as paracomplete and paraconsistent values, respectively.

Interestingly, some philosophers thought that both the paracomplete and the paraconsistent approach to pathological phenomena had something interesting to say, thus favoring a logical framework that allows both for truth-value gaps and for truth-value gluts. In other words, they suggested that work with transparent theories of truth is better conducted with an underlying four-valued logic counting with the classical truth-values and two additional non-classical values: one representing a gap, and one representing a glut-i.e. a paracomplete, and a paraconsistent value. A case for such four-valued approaches is made, for instance, in [74] by Albert Visser ${ }^{7}$

One attractive feature of four-valued logic for the study of the Liar Paradox is the possibility of making certain intuitive distinctions within one single model. Consider the Liar and the [Truth-Teller] (...) The intuitive idea here is that the Liar must be true, must be false; the [Truth-Teller] need not be true, need not be false. [74, p. $182]$

Thus, pace Visser, given the Liar must be true and must also be false, it should be both-trueand-false, i.e. a glut. Moreover, given the Truth-Teller need not be true and need not be false, it should be neither-true-nor-false, i.e. a gap. Additionally, it can clearly be read from Visser's quote, that he not only proposed an underlying four-valued logic with gaps and gluts for transparent theories of truth, but he went even further with his proposal. He claims that all paradoxical sentences should be taken as truth-value gluts, and that all hypodoxical sentences should be treated as truth-value gaps. ${ }^{8}$

However, this looks like a rather unnecessary decision, more than anything if we instructively counter Visser's account with the way he describes Kripke's perspective

\footnotetext{
${ }^{6}$ Meaning that the truth-value of the negation of a sentence that is both-true-and-false is, also, both-true-andfalse, and that the truth-value of the negation of a sentence that is neither-true-nor-false is, also, neither-truenor-false.

${ }^{7}$ Notice that Visser calls the sentence $\tau$ The Samesayer and we call it The Truth-Teller, whence the slight modification in Visser's quote.

${ }^{8}$ We should also comment on the fact that these observations led Visser to adopt Belnap-Dunn's four-valued logic FDE (cf. [29]) to model his ideas, i.e. a non-infectious four-valued logic according to Definition 3.1 below, whereas we will be focusing on infectious four-valued logics in what follows.
} 
For Kripke the Liar cannot be true, cannot be false; the [Truth-Teller] can be true, can be false. [74, p. 182]

Thus, given the Liar cannot be true and cannot be false, it should be neither-true-nor-false, i.e. a gap. Moreover, given the Truth-Teller can be true and can be false, it should be both-trueand-false, i.e. a glut. ${ }^{9}$ Furthermore, this alternative path seems to suggest that not only will it be reasonable to work with an underlying four-valued logic with gaps and gluts for transparent theories of truth, but something else. Namely, that all paradoxical sentences should be taken as truth-value gaps, and that all hypodoxical sentences should be treated as truth-value gluts.

As an additional option, one could also argue that given the alternatives above, it might be reasonable to sometimes treat paradoxes as gaps, and sometimes as gluts, while it also might be reasonable to sometimes treat hypodoxes as gaps, and sometimes as gluts. This will also make the case for four-valued approaches, although in this case no uniform policy for paradoxes and hypodoxes is assumed. ${ }^{10}$

In any case, if someone were to hold one of the previously summarized views - to the extent that some pathological sentences constitute truth-value gaps while some other represent truthvalues gluts - it would be sensible enough for her to adopt a four-valued logic counting with both paracomplete and paraconsistent values. In what follows, we will be exploring some four-valued systems that could be used for this purpose.

\subsection{Why infectious logics?}

Going back to the paracomplete or paraconsistent choice, it should be remarked that adopting a paracomplete or a paraconsistent view towards pathological sentences is not saying much about how pathological sentences behave, logically speaking. For there still remains the question of how do pathological sentences interact with non-pathological sentences. That is, to answer the question: is the conjunction or the disjunction of a non-pathological sentence and a pathological sentence pathological or not?

More specifically, assume we have two pathological sentences $A$ and $B$ such that our favored philosophical understanding renders $A$ as a truth-value gap and $B$ as truth-value glut. A legitimate question to ask, then, is: how should the following sentences be regarded?

Donald Trump won the 2016 Presidential Election of the USA or $A$

Hillary Clinton won the 2016 Presidential Election of the USA and $B$

Let us begin with sentence (1). Should (1) be considered as a true-and-not-false (i.e. just true) disjunction? Field and Bochvar, although both members of the paracomplete tradition, have adopted logical frameworks which incarnate radically different answers to this question. While, Field's approach in [37] entails that it should be, Bochvar's approach in [13] entails that it should not be.

In a nutshell, Field embraces a view on disjunctions that complies with the classical understanding of its truth and falsity conditions, according to which a disjunction is true if and only if it has at least one true disjunct, and is false if and only if it has two false disjuncts. Given this

\footnotetext{
${ }^{9}$ Let us clarify that we are not claiming that if a given sentence can be true and also can be false, then this is a good reason for treating it as a truth-value glut. (For, primarily, propositional letters share this feature with hypodoxical sentences, but we do not by any means support the idea that propositional letters should be regarded as truth-value gluts) What we claim, instead, is that if a given pathological sentence can be true and also can be false, then this is a plausible reason for treating such pathological sentences as a truth-value glut. We highlight, nevertheless, that we do not adopt neither this nor Visser's view, but remain open to both takes on this issue.

${ }^{10}$ This seems to be the option favored by Restall in [68].
} 
approach, sentence (1) has a true disjunct and must, therefore, be true. Moreover, it is not the case that it has two false disjuncts and, thus, it must be not false. This would render sentence (1) as true-and-not-false, i.e. just true.

Meanwhile, Bochvar adopts a view that can be depicted as modifying the truth-condition for disjunctions, saying that: a disjunction is true if and only if it has at least one true disjunct and none of its disjuncts is pathological. Since Bochvar is located within the paracomplete tradition, this can be rephrased as: a disjunction is true if and only if it has at least one true disjunct and none of its disjuncts is a truth-value gap.

Given this approach, sentence (1) has a gappy disjunct and, therefore, should be not true. Moreover, by the above it should also be not false. This, finally, would render sentence (1) as neither-true-nor-false, i.e. a truth-value gap itself. Notice, furthermore, that the outcome of this is that the inference commonly regarded as Addition or $\vee$-Introduction, i.e. $A \vDash A \vee B$, would be deemed as invalid.

Let us now move to sentence (2). Should (2) be considered as a false-and-not-true (i.e. just false) conjunction? Working in the paraconsistent tradition, Priest has adopted e.g. in [56] a logical framework which incarnates a positive answer to this question, but interestingly no other member of this tradition has put forward a logical framework that incarnates a negative answer to it-although Roberto Ciuni discusses in [19] an interpretation of Paraconsistent Weak Kleene logic (cf. [20]) along those lines.

Roughly, Priest embraces a view on conjunctions that complies with the classical understanding of its truth and falsity conditions, according to which a conjunction is true if and only if it has two true conjuncts, and is false if it has at least one false conjunct. Given this approach, sentence (2) has a false conjunct and must, therefore, be false. Moreover, it is not the case that it has two true conjuncts and, thus, it must be not true. This would render sentence (2) as false-and-not-true, i.e. just false.

Meanwhile, Ciuni proposed to understand conjunction in Paraconsistent Weak Kleene logic as being characterized by a modification of the truth-condition for conjunctions, saying that: a conjunction is true if and only if it has two true conjuncts or one of its conjuncts is pathological. Since he understands this logic as located within the paraconsistent tradition, he suggested to rephrase the above as: a conjunction is true if and only if it has two true conjuncts or some of its conjuncts is a truth-value glut. ${ }^{11}$

Given this approach, sentence (2) has a glutty conjunct and, therefore, should be true. Moreover, by the above it should also be false. This, finally, renders sentence (2) as both-trueand-false, i.e. a truth-value glut itself. Notice, furthermore, that the outcome of this is that the inference commonly regarded as Simplification or $\wedge$-Elimination, i.e. $A \wedge B \vDash A$, would be deemed invalid.

The plethora of different positions drawn above can be precisely summarized by saying that while some consider the semantic pathology suffered by paradoxical and hypodoxical sentences to be infectious, others think it is non-infectious. In more formal terms, we would say that while some consider the non-classical paracomplete or paraconsistent values to be infectious over the classical values, others think they should be non-infectious.

Of course, when we have different kind of pathological sentences (i.e. paradoxes and hypodoxes) being treated differently, this assessment must become more complex. Thus, we might

\footnotetext{
${ }^{11}$ As mentioned by an anonymous reviewer, the interpretation sketched (but not endorsed) in [19] seems less motivated than Priest's, which belongs to a well-developed tradition in paraconsistency. Moreover, we agree with the reviewer that, in fact, there is still a need for a better story regarding $\mathbf{P W K}$ - although a recent discussion concerning the reasonability of conjunction and disjunction as featured in infectious logics can be found in [51]. However, important as it is, in this paper we take the discussion as presented in the relevant literature, and leave deeper considerations about the philosophical motivations, e.g. for Paraconsistent Weak Kleene, for further research.
} 
settle for one of the following options:

1. the semantic pathology suffered by hypodoxical sentences is infectious, while that suffered by paradoxical sentences is not,

2. the semantic pathology suffered by paradoxical sentences is infectious, while that suffered by hypodoxical sentences is not,

3. the semantic pathology suffered by both paradoxical and hypodoxical sentences is infectious, but hypodoxes suffer from a pathology that is more infectious than paradoxes,

4. the semantic pathology suffered by both paradoxical and hypodoxical sentences is infectious, but paradoxes suffer from a pathology that is more infectious than hypodoxes.

Now, if we are willing to deal with pathological sentences within a four-valued logic - drawing inspiration from the ideas discussed in the previous section - then this quadripartite classification will lead us to consider theories of truth built on top of four-valued logics where, in terms of Definition 3.1 below, one of the following holds: ${ }^{12}$

- the paracomplete value is infectious and the paraconsistent value is not (this case being represented by the logic $\mathbf{S}_{\text {fde }}$ )

- the paraconsistent value is infectious and the paracomplete value is not (this case being represented by the logic $\mathbf{d} \mathbf{S}_{\text {fde }}$ )

- both the paracomplete and the paraconsistent value are infectious over the classical values, but the paracomplete value is infectious over the paraconsistent value (this case being represented by the logic $\mathbf{S}_{\text {fde }}^{w}$ )

- both the paracomplete and the paraconsistent value are infectious over the classical values, but the paraconsistent value is infectious over the paracomplete value (this case being represented by the logic $\mathbf{d} \mathbf{S}_{\text {fde }}^{w}$ )

We shall mention that, on the one hand, the logic $\mathbf{S}_{\mathrm{fde}}$ is the first-degree fragment of the logic $\mathbf{S}$ due to Harry Deutsch in [26], while on the other hand the logics $\mathbf{d} \mathbf{S}_{\mathrm{fde}}, \mathbf{S}_{\mathrm{fde}}^{w}$ and $\mathbf{d} \mathbf{S}_{\mathrm{fde}}^{w}$ were introduced in [72] for the first time, although with different names. ${ }^{13}$ We will spell out these systems in Definitions 3.4 and 3.5 in the next section, though.

Finally, if we are asked which is the route we favor, we shall answer that none in particular. Our work here is exploratory. As Kripke says with regard to the various formal tools that he developed to deal with transparent theories of truth

Nor is it even my present purpose to make any firm recommendation (...) My purpose is rather to provide a family of flexible instruments which can be explored simultaneously and whose fertility and consonance with intuition can be checked. [46, p. $712]$

Instead of favoring one approach, our aim is to argue that four-valued theories of truth have the appealing feature of allowing to make differences between different kind of pathological sentences. We will be neutral about the various logical basis that we will present, hoping that the reader will consider which of the philosophical reasons presented here makes her decide for one of the suggested options.

\footnotetext{
${ }^{12}$ The reader should keep in mind that there is no necessary correlation with the options 1 to 4 above and the order in which the logics are presented. For, as we discussed previously, one could either want to evaluate paradoxical sentences as gaps or gluts, and the same holds for hypodoxical sentences.

${ }^{13}$ In the context of [72] the logics $\mathbf{d} \mathbf{S}_{\text {fde }}, \mathbf{S}_{\text {fde }}^{w}$ and $\mathbf{d} \mathbf{S}_{\text {fde }}^{w}$ were, respectively, referred to as $\mathbf{L}_{\mathbf{n b}} \mathbf{L}_{\mathbf{b}} \mathbf{L}_{\mathbf{e}}$ and $\mathbf{L}_{\mathbf{e b}}$.
} 


\section{Theories of truth based on infectious logics}

To carry out our study, we start with a base language $\mathcal{L}$ which is composed of a denumerable set of propositional variables $\operatorname{Var}$ and a set of connectives $C=\{\neg, \wedge, \vee\}$, letting the set of formulae recursively defined in this language be the set $\mathbf{F m l}{ }_{\mathcal{L}}$. We denote, as usual, formulae with capital Roman letters $A, B, C$, etc., and set of formulae of the language by capital Greek letters $\Gamma, \Delta, \Sigma$, etc. A logic $\mathbf{L}$ is a pair $\left\langle\mathbf{F m l}{ }_{\mathcal{L}}, \vdash_{\mathbf{L}}\right\rangle$, where $\vdash_{\mathbf{L}} \subseteq \wp\left(\mathbf{F m l}_{\mathcal{L}}\right) \times \mathbf{F m l}_{\mathcal{L}}$ is a substitution-invariant consequence relation.

In turn, by a matrix logic $\mathbf{L}$ we understand a pair $\left\langle\mathbf{F m l}_{\mathcal{L}}, \vDash \mathcal{M}\right\rangle$ where $\vDash^{\mathcal{M}}$ is a substitutioninvariant consequence relation induced by a logical matrix $\mathcal{M}=\langle\mathcal{V}, \mathcal{D}, \mathcal{O}\rangle$, such that $\langle\mathcal{V}, \mathcal{O}\rangle$ is an algebra of the same similarity type as $\mathcal{L}$, with universe $\mathcal{V}$ and a set of operations $\mathcal{O}$, and $\mathcal{D} \subseteq \mathcal{V} .{ }^{14}$ As usual, an $\mathcal{M}$-valuation $v$ is a homomorphism from $\mathbf{F m l}_{\mathcal{L}}$ to $\mathcal{V}$, for which we denote by $v[\Gamma]$ the set $\{v(B) \mid B \in \Gamma\}$, i.e. the image of $v$ under $\Gamma$. A logical matrix $\mathcal{M}=\langle\mathcal{V}, \mathcal{D}, \mathcal{O}\rangle$ induces a substitution-invariant consequence relation $\vDash^{\mathcal{M}}$ by letting

$$
\Gamma \vDash^{\mathcal{M}} A \Longleftrightarrow \text { for every } \mathcal{M} \text {-valuation } v \text {, if } v[\Gamma] \subseteq \mathcal{D} \text {, then } v(A) \in \mathcal{D}
$$

Moreover, since we want to deal with a truth predicate and this linguistic possibility often implies the rise of potentially pathological phenomena represented by e.g. self-referential sentences that lead to paradoxes, we will need formal devices to model these facts. Given the former requirement, we should have a symbol $\operatorname{Tr}$ for a truth predicate; given the latter requirement, we need the language containing the truth predicate to have some way to talk about its own expressions. This is the key to even have these pathological sentences around. ${ }^{15}$

The target of this paper are infectious logics, which are induced by certain matrix logics defined as follows. ${ }^{16}$

Definition 3.1. We say that a matrix logic $\mathbf{L}=\left\langle\mathbf{F m l}_{\mathcal{L}}, \models \mathcal{M}\right\rangle$ is infectious if and only if there is an element $\mathbf{x} \in \mathcal{V}$ such that for every $n$-ary operator $\diamond \in \mathcal{L}$ with an associated truth-function $f_{\mathcal{M}}^{\diamond} \in \mathcal{O}$ and for all $\mathbf{v}_{1}, \ldots, \mathbf{v}_{n} \in \mathcal{V}$ it holds that:

$$
\text { if } \mathbf{x} \in\left\{\mathbf{v}_{1}, \ldots, \mathbf{v}_{n}\right\} \text {, then } f_{\mathcal{M}}^{\diamond}\left(\mathbf{v}_{1}, \ldots, \mathbf{v}_{n}\right)=\mathbf{x}
$$

When it is clear from the context, we will refer to the distinctive truth-values of infectious logics as being themselves infectious values. Intuitively, infectious logics are motivated by the idea that 'one bad apple spoils the whole barrel'-although 'spoils' has a negative connotation, while infectious values and infectious logics do not necessarily share this negative appreciation. ${ }^{17}$

\footnotetext{
${ }^{14}$ Notice that $\mathcal{O}$ is a set that includes for every $n$-ary operator $\diamond$ in the language $\mathcal{L}$, a corresponding $n$-ary truth-function $f_{\mathcal{M}}^{\diamond}: \mathcal{V}^{n} \longrightarrow \mathcal{V}$. Moreover, when context allows it, we will sometimes identify the connectives themselves (which are linguistic items), with their corresponding truth-functions in a given matrix.

${ }^{15}$ Moreover, given our interest in formal theories of truth based on infectious logics, which are formulated using first-order languages, the notion of a semantic structure for many-valued logics has to be well-defined for these languages too. Nevertheless, since we will not be making use of quantifiers or predicates other than the truth predicate, it is enough to restrict ourselves here to the exposition of more simple semantic structures, which suffice for the purpose of the ongoing discussion. We defer the reader, though, to the precise definition of a semantic structure for many-valued first-order logics given e.g. by Graham Priest in [60, chapter 21].

${ }^{16}$ The definition is inspired by [44], where some infectious logics are scrutinized under the magnifying glass of power algebras and power matrices. A similar definition might be found in [35]. This definition is extended to cover non-deterministic matrices in [72], where also alternative notions of infectiousness related to non-deterministic semantics are discussed.

${ }^{17} \mathrm{By}$ this we mean that systems referred to as infectious logics in the recent literature (e.g. in [35], [72], [51]) do not need to be considered undesirable systems, for they can be proven to be interesting, useful and illuminating in many respects - which we do not have space to review here, for it will take us too far afield. We do, nevertheless, share the opinion of an anonymous referee, who called to attention that referring to something as "infectious" clearly does carry a disease metaphor and sounds negative. Whence, perhaps it would be better to say that
} 
This feature has been dubbed by Lennar Åvist as 'the doctrine of the predominance of the atheoretical element' [1], but is more often referred as a sort of contamination, infectiousness, or absorbing nature for obvious reasons.

Finally, throughout this paper, when we refer to infectious logics, we will be actually meaning 'infectious logics that are sublogics of classical logic', i.e. matrix logics where the classical truthvalues behave normally and the characteristic infectious value is non-classical. We now proceed to define some of these. We start, however, with two paradigmatically non-infectious logics and then proceed to consider infectious cases.

Definition 3.2. Strong Kleene logic $\mathbf{K}_{\mathbf{3}}$ and the Logic of Paradox $\mathbf{L P}$ are the three-valued logics induced, respectively, by the matrix $\left\langle\mathcal{V}_{\mathbf{K}_{3}}, \mathcal{D}_{\mathbf{K}_{3}}, \mathcal{O}_{\mathbf{K}_{3}}\right\rangle$ and by the matrix $\left\langle\mathcal{V}_{\mathbf{L P}}, \mathcal{D}_{\mathbf{L P}}, \mathcal{O}_{\mathbf{L P}}\right\rangle$, where $\mathcal{V}_{\mathbf{K}_{3}}=\mathcal{V}_{\mathbf{L P}}=\{\mathbf{t}, \mathbf{i}, \mathbf{f}\}, \mathcal{D}_{\mathbf{K}_{3}}=\{\mathbf{t}\}, \mathcal{D}_{\mathbf{L P}}=\{\mathbf{t}, \mathbf{i}\}$ and $\mathcal{O}_{\mathbf{K}_{3}}=\mathcal{O}_{\mathbf{L P}}=\left\{f_{\mathbf{K}_{3} / \mathbf{L P}}^{\neg}, f_{\mathbf{K}_{3} / \mathbf{L P}}^{\wedge}, f_{\mathbf{K}_{3} / \mathbf{L P}}^{\vee}\right\}$ where these truth-functions are as follows

\begin{tabular}{|c|c|c|c|c|c|c|c|c|c|}
\hline & $f_{\mathbf{K}_{3} / \mathbf{L P}}^{\neg}$ & $f_{\mathbf{K}_{3} / \mathbf{L P}}^{\wedge}$ & $\mathbf{t}$ & i & f & $f_{\mathbf{K}_{\mathbf{3}} / \mathbf{L P}}^{\vee}$ & t & i & f \\
\hline $\mathrm{t}$ & $f$ & $\mathbf{t}$ & $t$ & $\mathrm{i}$ & $f$ & $t$ & $\mathrm{t}$ & $\mathrm{t}$ & $\mathrm{t}$ \\
\hline i & $\mathrm{i}$ & $\mathrm{i}$ & $\mathrm{i}$ & $\mathrm{i}$ & f & $\mathrm{i}$ & $t$ & $\mathrm{i}$ & $\mathbf{i}$ \\
\hline f & $\mathbf{t}$ & f & f & f & f & f & $t$ & i & f \\
\hline
\end{tabular}

Definition 3.3. (Paracomplete) Weak Kleene logic $\mathbf{K}_{3}^{\mathbf{w}}$ and Paraconsistent Weak Kleene logic $\mathbf{P W K}$ are the three-valued logics induced, respectively, by the matrix $\left\langle\mathcal{V}_{\mathbf{K}_{3}^{\mathrm{w}}}, \mathcal{D}_{\mathbf{K}_{3}^{\mathrm{w}}}, \mathcal{O}_{\mathbf{K}_{3}^{\mathrm{w}}}\right\rangle$ and by the matrix $\left\langle\mathcal{V}_{\mathbf{P W K}}, \mathcal{D}_{\mathbf{P W K}}, \mathcal{O}_{\mathbf{P W K}}\right\rangle$, where $\mathcal{V}_{\mathbf{K}_{3}^{\mathbf{w}}}=\mathcal{V}_{\mathbf{P W K}}=\{\mathbf{t}, \mathbf{u}, \mathbf{f}\}, \mathcal{D}_{\mathbf{K}_{3}^{\mathrm{w}}}=\{\mathbf{t}\}, \mathcal{D}_{\mathbf{P W K}}=$ $\{\mathbf{t}, \mathbf{u}\}$ and $\mathcal{O}_{\mathbf{K}_{3}^{\mathbf{w}}}=\mathcal{O}_{\mathbf{P W K}}=\left\{f_{\mathbf{K}_{3}^{\mathbf{w}} / \mathbf{P W K}}^{\neg}, f_{\mathbf{K}_{3}^{\mathbf{w}} / \mathbf{P W K}}^{\wedge}, f_{\mathbf{K}_{3}^{\mathbf{w}} / \mathbf{P W K}}^{\vee}\right\}$ where these truth-functions are as follows

\begin{tabular}{|c|c|c|c|c|c|c|c|c|c|}
\hline & $f_{\mathbf{K}}^{\neg}{ }_{\mathbf{3}}^{\mathbf{w}} / \mathbf{P W K}$ & $f_{\mathbf{K}_{3}^{\mathbf{w}} / \mathbf{P W K}}^{\wedge}$ & $\mathrm{t}$ & $\mathbf{u}$ & f & $f_{\mathbf{K}_{3}^{\mathbf{w}} / \mathbf{P W K}}^{\vee}$ & $\mathrm{t}$ & $\mathbf{u}$ & f \\
\hline $\bar{t}$ & $\mathrm{f}$ & $\mathrm{t}$ & $t$ & $\mathbf{u}$ & $f$ & $\mathrm{t}$ & $\mathrm{t}$ & $\mathbf{u}$ & $\mathrm{t}$ \\
\hline $\mathbf{u}$ & $\mathbf{u}$ & $\mathbf{u}$ & $\mathbf{u}$ & $\mathbf{u}$ & $\mathbf{u}$ & $\mathbf{u}$ & $\mathbf{u}$ & $\mathbf{u}$ & $\mathbf{u}$ \\
\hline f & $\mathbf{t}$ & f & $\mathrm{f}$ & $\mathbf{u}$ & f & f & $\mathbf{t}$ & $\mathbf{u}$ & f \\
\hline
\end{tabular}

Definition 3.4. The logics $\mathbf{S}_{\mathrm{fde}}$ and its dual $\mathbf{d S}_{\mathrm{fde}}$ are induced by the matrices $\left\langle\mathcal{V}_{\mathbf{S}_{\mathrm{fde}}}, \mathcal{D}_{\mathrm{S}_{\mathrm{fde}}}, \mathcal{O}_{\mathbf{S}_{\mathrm{fde}}}\right\rangle$ and $\left\langle\mathcal{V}_{\mathrm{dS}_{\mathrm{fde}}}, \mathcal{D}_{\mathrm{dS}_{\mathrm{fde}}}, \mathcal{O}_{\mathrm{dS}_{\mathrm{fde}}}\right\rangle$, where $\mathcal{V}_{\mathrm{dS}_{\mathrm{fde}}}=\mathcal{V}_{\mathbf{S}_{\mathrm{fde}}}=\{\mathbf{t}, \mathbf{i}, \mathbf{u}, \mathbf{f}\}, \mathcal{D}_{\mathrm{S}_{\mathrm{fde}}}=\{\mathbf{t}, \mathbf{i}\}, \mathcal{D}_{\mathrm{dS}_{\mathrm{fde}}}=\{\mathbf{t}, \mathbf{u}\}$, $\mathcal{O}_{\mathbf{S}_{\mathrm{fde}}}=\mathcal{O}_{\mathbf{d S}_{\mathrm{fde}}}=\left\{f_{\mathbf{S}_{\mathrm{fde}} / \mathbf{d S}_{\mathrm{fde}}}, f_{\mathbf{S}_{\mathrm{fde}} / \mathbf{d S}_{\mathrm{fde}}}^{\wedge} f_{\mathbf{S}_{\mathrm{fde}} / \mathbf{d S}_{\mathrm{fde}}}^{\vee}\right\}$ where these truth-functions are as follows

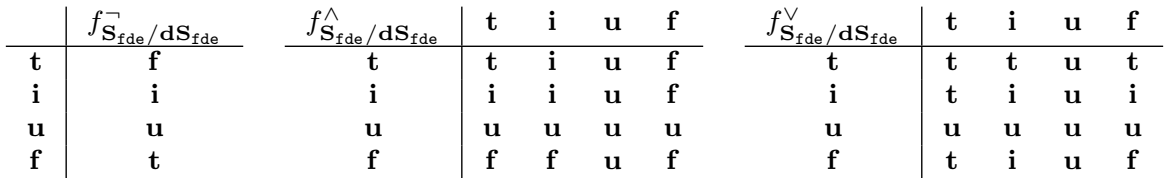

Definition 3.5. The logics $\mathbf{S}_{\mathrm{fde}}^{w}$ and its dual $\mathbf{d S}_{\mathrm{fde}}^{w}$ are induced by the matrices $\left\langle\mathcal{V}_{\mathbf{S}_{\mathrm{fde}}^{w}}, \mathcal{D}_{\mathbf{S}_{\mathrm{fde}}}^{w}, \mathcal{O}_{\mathbf{S}_{\mathrm{fde}}^{w}}\right\rangle$ and $\left\langle\mathcal{V}_{\mathbf{d S}_{\mathrm{fde}}^{w}}^{w}, \mathcal{D}_{\mathbf{d S}_{\mathrm{fde}}^{w}}^{w}, \mathcal{O}_{\mathbf{d S}_{\mathrm{fde}}^{w}}^{w}\right\rangle$, where $\mathcal{V}_{\mathbf{S}_{\text {fde }}^{w}}^{w}=\mathcal{V}_{\mathbf{d S}_{\mathrm{fde}}^{w}}^{w}=\left\{\mathbf{t}, \mathbf{u}_{1}, \mathbf{u}_{2}, \mathbf{f}\right\}, \mathcal{D}_{\mathbf{S}_{\text {fde }}^{w}}^{w}=\left\{\mathbf{t}, \mathbf{u}_{1}\right\}, \mathcal{D}_{\mathbf{d S}_{\text {fde }}}^{w}=$ $\left\{\mathbf{t}, \mathbf{u}_{2}\right\}, \mathcal{O}_{\mathbf{S}_{\mathrm{fde}}}^{w}=\mathcal{O}_{\mathbf{d S}_{\mathrm{fde}}^{w}}^{w}=\left\{f_{\mathbf{S}_{\mathrm{fde}}^{w} / \mathbf{d S}_{\mathrm{fde}}^{w}}^{\neg} f_{\mathbf{S}_{\mathrm{fde}}^{w} / \mathbf{d S}_{\mathrm{fde}}^{w}}^{\wedge} f_{\mathbf{S}_{\mathrm{fde}}^{w} / \mathbf{d S}_{\mathrm{fde}}^{w}}^{\vee}\right\}$ where these truth-functions are as follows

\begin{tabular}{|c|c|c|c|c|c|c|c|c|c|c|c|}
\hline & $f_{\mathbf{S}_{\mathrm{fde}}^{w} / \mathbf{d S}_{\mathrm{fde}}^{w}}^{\sim}$ & $f_{\mathbf{S}_{\mathrm{fde}}^{w} / \mathbf{d} \mathbf{S}_{\mathrm{fde}}^{w}}^{\wedge}$ & $\mathbf{t}$ & $\mathbf{u}_{1}$ & $\mathbf{u}_{2}$ & f & $f_{\mathbf{S}_{\text {fde }}^{w} / \mathbf{d S}_{\text {fde }}^{w}}^{\vee}$ & $\mathbf{t}$ & $\mathbf{u}_{1}$ & $\mathbf{u}_{2}$ & $\mathbf{f}$ \\
\hline $\mathbf{t}$ & f & $\mathbf{t}$ & $\mathbf{t}$ & $\mathbf{u}_{1}$ & $\mathbf{u}_{2}$ & f & $\mathbf{t}$ & $\mathbf{t}$ & $\mathbf{u}_{1}$ & $\mathbf{u}_{2}$ & $\mathbf{t}$ \\
\hline $\mathbf{u}_{1}$ & $\mathbf{u}_{1}$ & $\mathbf{u}_{1}$ & $\mathbf{u}_{1}$ & $\mathbf{u}_{1}$ & $\mathbf{u}_{2}$ & $\mathbf{u}_{1}$ & $\mathbf{u}_{1}$ & $\mathbf{u}_{1}$ & $\mathbf{u}_{1}$ & $\mathbf{u}_{2}$ & $\mathbf{u}_{1}$ \\
\hline $\mathbf{u}_{2}$ & $\mathbf{u}_{2}$ & $\mathbf{u}_{2}$ & $\mathbf{u}_{2}$ & $\mathbf{u}_{2}$ & $\mathbf{u}_{2}$ & $\mathbf{u}_{2}$ & $\mathbf{u}_{2}$ & $\mathbf{u}_{2}$ & $\mathbf{u}_{2}$ & $\mathbf{u}_{2}$ & $\mathbf{u}_{2}$ \\
\hline $\mathbf{f}$ & $\mathbf{t}$ & $\mathbf{f}$ & $\mathbf{f}$ & $\mathbf{u}_{1}$ & $\mathbf{u}_{2}$ & $\mathbf{f}$ & $\mathbf{f}$ & $\mathbf{t}$ & $\mathbf{u}_{1}$ & $\mathbf{u}_{2}$ & $\mathbf{f}$ \\
\hline
\end{tabular}

such systems count with an absorbing or absorbent element, as Humberstone does in [44, p. 20]. Neverthelessalthough we are thankful to the anonymous referee for pointing this out - in the context of the present work we will follow the current terminology, for the sake of keeping things simpler. 
Remark 3.6. There is a clear inclusion between the four-and the three-valued systems presented above, such that $\mathbf{S}_{\mathrm{fde}}, \mathbf{S}_{\mathrm{fde}}^{w}$, and $\mathbf{d} \mathbf{S}_{\mathrm{fde}}^{w}$ are subsystems of $\mathbf{K}_{\mathbf{3}}^{\mathbf{w}}$, while $\mathbf{d} \mathbf{S}_{\mathrm{fde}}, \mathbf{S}_{\mathrm{fde}}^{w}$, and $\mathbf{d} \mathbf{S}_{\mathrm{fde}}^{w}$ are subsystems of $\mathbf{P W K}$, in addition to $\mathbf{S}_{\mathrm{fde}}$ being also a subsystem of $\mathbf{L P}$, and $\mathbf{d} \mathbf{S}_{\mathrm{fde}}$ being also a subsystem of $\mathbf{K}_{\mathbf{3}}$. These facts are easily established by looking at their matrices and noticing that (provided an intuitive mapping) the set of valuations from the corresponding sublogics is a superset of the set of valuations of the respective logics.

Remark 3.7. We notice the duality between the previously mentioned pair of logics by letting $\Sigma\urcorner=\{\neg C \mid C \in \Sigma\}$, for any given set of sentences $\Sigma$. Thus, for $(x, y)$ or $(y, x)$ $\in\left\{\left(\mathbf{K}_{\mathbf{3}}, \mathbf{L P}\right),\left(\mathbf{K}_{\mathbf{3}}^{\mathbf{w}}, \mathbf{P W K}\right),\left(\mathbf{S}_{\mathrm{fde}}, \mathbf{d S}_{\mathrm{fde}}\right),\left(\mathbf{S}_{\mathrm{fde}}^{w}, \mathbf{d} \mathbf{S}_{\text {fde }}^{w}\right)\right\}$ we can establish the following, which we leave to the reader to straightforwardly check.

$$
\left.\left.\Gamma \vDash_{x} \Delta \text { iff } \Delta\right\urcorner \vDash_{y} \Gamma\right\urcorner
$$

We should highlight that, in addition to the previously mentioned weak nature of disjunction and conjunction in infectious logics, we can indeed provide a few examples of the inferential failures provoked by the peculiar nature of these logical connectives in these contexts.

\begin{tabular}{c|cccccc} 
& $\mathbf{K}_{\mathbf{3}}^{\mathbf{w}}$ & $\mathbf{P W K}$ & $\mathbf{d S}_{\text {fde }}$ & $\mathbf{S}_{\text {fde }}$ & $\mathbf{S}_{\text {fde }}^{w}$ & $\mathbf{d S}_{\text {fde }}^{w}$ \\
\hline$B \vDash A \vee \neg A$ & $\times$ & $\checkmark$ & $\times$ & $\times$ & $\times$ & $\times$ \\
$A \vDash A \vee B$ & $\times$ & $\checkmark$ & $\checkmark$ & $\times$ & $\times$ & $\times$ \\
$A \wedge \neg A \vDash(A \wedge \neg A) \vee B$ & $\checkmark$ & $\checkmark$ & $\checkmark$ & $\times$ & $\times$ & $\checkmark$ \\
$A \wedge \neg A \vDash B$ & $\checkmark$ & $\times$ & $\times$ & $\times$ & $\times$ & $\times$ \\
$A \wedge B \vDash A$ & $\checkmark$ & $\times$ & $\times$ & $\checkmark$ & $\times$ & $\times$ \\
$(A \vee \neg A) \wedge B \vDash A \vee \neg A$ & $\checkmark$ & $\checkmark$ & $\times$ & $\checkmark$ & $\checkmark$ & $\times$
\end{tabular}

Let us mention a few remarks with regard to these inferential facts. That $\vee$-Introduction, i.e. $A \vDash A \vee B$, fails for infectious paracomplete logics is interpreted by Ferguson in [33] as a sign that disjunction has a conjunctive nature in this kind of logics, by means of which he refers to the fact that a disjunction is true if and only if at least one of its disjuncts is true and none of the disjuncts is a truth-value gap - in accordance with our exposition in the previous sections. Moreover, that a restricted version of $\vee$-Introduction, i.e. $A \wedge \neg A \vDash(A \wedge \neg A) \vee B$, is invalid in $\mathbf{S}_{\mathrm{fde}}$ and $\mathbf{S}_{\mathrm{fde}}^{w}$ is precisely explained by the fact that these logics allow contradictions to be true. Whence, even if $A \wedge \neg A$ is true, if $B$ is a truth-value gap, then $(A \wedge \neg A) \vee B$ must be a truth-value gap according to the infectious paracomplete doctrine.

Interestingly, that this inference is valid in $\mathbf{d} \mathbf{S}_{\text {fde }}^{w}$ is explained by the fact that for this logic the semantic pathology suffered by glutty sentences is more infectious than the semantic pathology suffered by gappy sentences. More specifically, if $A \wedge \neg A$ is true, it must be a glut. Thus, $(A \wedge \neg A) \vee B$ will end up being a glut, i.e. a true sentence itself - even if $B$ happens to be a gappy sentence.

Analogously, that $\wedge$-Elimination, i.e. $A \wedge B \vDash A$, fails for infectious paraconsistent logics is interpreted by Ciuni in [19] as a sign that conjunction has a disjunctive nature in this kind of logics, by means of which he refers to the fact that a conjunction is true if and only if both of its conjuncts are true or some of the disjuncts is a truth-value glut. Moreover, that a restricted version of $\wedge$-Elimination, i.e. $(A \vee \neg A) \wedge B \vDash A \vee \neg A$, is invalid in $\mathbf{d S}_{\text {fde }}$ and $\mathbf{d S}_{\text {fde }}^{w}$ is precisely explained by the fact that these logics allow sentences of the form $A \vee \neg A$ to be neither true nor false. Whence, even if $A \vee \neg A$ is neither true nor false, if $B$ is a truth-value glut, then $(A \vee \neg A) \wedge B$ must be a a truth-value glut according to the infectious paraconsistent doctrine.

Remarkably, that this inference holds in $\mathbf{S}_{\text {fde }}^{w}$ is explained by the fact that for this logic the semantic pathology suffered by gappy sentences is more infectious than the semantic pathology suffered by glutty sentences. More specifically, if $A \vee \neg A$ is not true, it must be a gap. Thus, $(A \vee \neg A) \wedge B$ will end up being a gap, i.e. a sentence that is not true - even if $B$ happens to be a glutty sentence. 
Finally, we need to clarify that we are after theories of truth that are transparent, i.e. theories such that for every sentence $A$ whose name is $\langle A\rangle$, and for all valuations $v$,

$$
v(A)=v(\operatorname{Tr}(\langle A\rangle))
$$

For a given logic $\mathbf{L}$ of the previously defined systems, we refer to the theories of truth formulated in these languages as LTT. ${ }^{18}$ To close this section we cite some important non-triviality results for this logics. In the upcoming sections we will endow these logics and their corresponding transparent theories of truth with appropriate and philosophically justified proof-theoretic means.

Fact $3.8([46])$. The transparent theory of truth $\mathbf{K}_{\mathbf{3}} \mathbf{T T}$ is non-trivial

Fact 3.9 ([58], [12]). The transparent theory of truth LPTT is non-trivial

Fact 3.10 ([41], [40]). The transparent theory of truth $\mathbf{K}_{\mathbf{3}}^{\mathbf{w}} \mathbf{T} \mathbf{T}$ is non-trivial

Corollary 3.11 ([41], [40]). The transparent theory of truth PWKTT is non-trivial

Proof. Follows from the duality of $\mathbf{K}_{\mathbf{3}}^{\mathrm{w}} \mathbf{T T}$ and PWKTT.

Corollary 3.12. The transparent theories of truth $\mathbf{S}_{\text {fde }} \mathbf{T T}, \mathbf{d} \mathbf{S}_{\text {fde }} \mathbf{T T}, \mathbf{S}_{\text {fde }}^{w} \mathbf{T T}, \mathbf{d S}_{\text {fde }}^{w} \mathbf{T T}$ are non-trivial

Proof. Follows from the fact that they are subsystems of the non-trivial theories of truth $\mathbf{K}_{\mathbf{3}} \mathbf{T T}$,

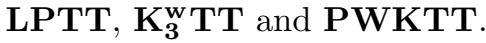

Having reviewed these results, we now proceed to the proof-theoretic examination of these formal theories of truth. In what follows we will prove some limitative results regarding the availability of ordinary two-sided sequent calculi for these logics and their corresponding theories of truth. After that, we will show that it is possible to provide four-sided sequent calculi for all the target systems alongside with discussing some philosophical reasons to favor these calculi over other plausible alternatives.

\section{Limitative results regarding standard Gentzen calculi for infectious logics}

In this section we will present some limitative results with respect to the proof theory of the systems introduced above. In particular, in [14] the authors prove that a large family of paraconsistent logics, including paraconsistent infectious logics, cannot have any sound and complete standard sequent calculus. ${ }^{19}$ In that paper, the authors define standard sequent calculus in the following way:

\footnotetext{
${ }^{18}$ More formally, when working with first-order systems, languages will be constructed as follows. First, we start with a base first-order language $\mathcal{L}$ which we extend this as follows. We obtain the language $\mathcal{L}_{T r}$ by means of adding a unary predicate symbol $\operatorname{Tr}$ to $\mathcal{L}$ intended to be interpreted later as the truth predicate. In this step, we also add a set of distinguished constants $\mathcal{B}=\left\{b_{1}, b_{2}, b_{3}, \ldots\right\}$. We require that all semantic structures interpreting this language are such that the denotation of each of these constants is given by a function $f_{1}$ such that $f_{1}: \mathcal{B} \longrightarrow \mathbf{F m l}_{\mathcal{L}_{T r}}$, where as usual $\mathbf{F m l}_{\mathcal{L}_{T r}}$ stands for the set of $\mathcal{L}_{T r}$ formulae. To ease the notation, we will write $\langle A\rangle$ for the distinguished name $c \in \mathcal{B}$ such that $f_{1}(c)=A$. We borrow this way of getting self-reference from $[69]$.

${ }^{19} \mathrm{~A}$ sequent is an ordered pair of multisets of formulas, represented as $\Gamma \Rightarrow \Delta$. Multisets are basically sets with repetitions. That is, a multiset is just like a set except for the fact that it is sensitive to the different occurrences of a member. In this sense, $\{A, A\}$ and $\{A\}$ are different multisets. The main reason for moving from sets to multisets is that, in Definition 4.1 taken from [14], the structural rule of Contraction is explicitly mentioned.
} 
Definition 4.1. ([14]) We say that a Gentzen calculus for a logic $\mathbf{L}$ is standard if it has the following properties:

1. Axioms shall be only of the form $A \Rightarrow A$ for any propositional variable $A$.

2. The premises of logical rules must contain only subformulas of the conclusion and each logical rule must introduce exactly one connective at a time.

3. Logical rules must have no linguistic restrictions.

4. Sequents shall be interpreted in the object language, that is: $\Gamma \Rightarrow \Delta$ means that the formula $\bigvee_{i=1}^{n} A_{i}$, with $A_{i} \in \Delta$ follows from $\bigwedge_{j=1}^{m} B_{m}$ with $B_{m} \in \Gamma$.

5. Only standard structural rules, i.e. Contraction, Weakening and Cut are (possibly) allowed. ${ }^{20}$

So, they prove the following two main relevant facts (from which we extract the pertinent corollaries):

Fact 4.2 ([14]). The logic PWK does not admit any sound and complete standard sequent calculus.

Fact 4.3 ([14]). The logic $\mathbf{L P}$ does not admit any sound and complete standard sequent calculus.

Fact 4.4. The logic $\mathbf{d S}_{\mathrm{fde}}$ does not admit any sound and complete standard sequent calculus.

Proof. It is straightforward to adapt the proof given in [14, pp. 5-6] for PWK. The general strategy is the one we dualize below, in order to prove Theorem 4.9. Basically, as we reproduce in Remark 4.6 and we dualize in Lemma 4.8, the authors in [14] show that any standard Gentzen calculus is such that the rules for left and right conjunction, i.e. $(L \wedge)$ and $(R \wedge)$, respectively, should have the following form (letting $P_{j}$, with $j=0, \ldots, m$ be possibly additional sequents):

$$
\frac{\Gamma, A, B \Rightarrow \Delta}{\Gamma, A \wedge B \Rightarrow \Delta} L \wedge \quad \frac{\Gamma \Rightarrow \Delta, A \quad \Gamma \Rightarrow \Delta, B \quad P_{j}, \text { with } j=0, \ldots, m}{\Gamma \Rightarrow A \wedge B, \Delta} R \wedge
$$

Then, it is necessary to prove that if the rule of left weakening $(L W)$ is admitted, the calculus is unsound - in fact, we follow a similar strategy in our proof of Remark 4.7. Finally, it is necessary to prove that if the rule of left weakening is not included in the calculus, the resulting system is incomplete. This is shown by noticing that given the axioms are only allowed to have propositional variables, then any derivation of the sequent $A \wedge B \Rightarrow A \wedge B$, must employ the rule $(L W)$ - again, as we advanced, a similar strategy is followed by us in proving Theorem 4.9, below.

Corollary 4.5. The transparent theories of truth $\mathbf{P W K T T}$, LPTT and $\mathbf{d S}_{\mathrm{fde}} \mathbf{T T}$ do not admit any sound and complete standard sequent calculus.

However, as far as we know, there is no similar results in the literature regarding paracomplete and, most importantly for us, paracomplete infectious logics. ${ }^{21}$ In view of that, here we will extend and generalize these results, taking some definitions and terminology from [14]. So, we will prove that it is not possible to obtain any sound and complete standard sequent calculus for the paracomplete infectious logic Weak Kleene $\mathbf{K}_{\mathbf{3}}^{\mathbf{w}}$, and neither is it for its sublogics, $\mathbf{S}_{\mathrm{fde}}$, $\mathbf{d} \mathbf{S}_{\mathrm{fde}}^{w}$, and $\mathbf{S}_{\mathrm{fde}}^{w}$. Lastly, once we prove this, it is easy to generalize these limitative results to $\mathbf{K}_{3}^{\mathbf{w} T T}, \mathbf{S}_{\text {fde }} \mathbf{T T}, \mathbf{d} \mathbf{S}_{\text {fde }}^{w} \mathbf{T T}$ and $\mathbf{S}_{\text {fde }}^{w} \mathbf{T T}$.

Thus, let $\mathbf{K}_{\mathbf{3}}^{\mathbf{w}}, \mathbf{S}_{\text {fde }}, \mathbf{d S}_{\text {fde }}^{w}, \mathbf{S}_{\text {fde }}^{w}$ be the logics we will work with. From Definition 4.1, we derive the following remarks:

\footnotetext{
${ }^{20}$ It is worth noting that other structural rules as associativity and exchange are assumed, since we are working with multisets.

${ }^{21}$ In [7] the authors show a similar limitative result, but regarding what they call canonical Gentzen calculus. However, we prove our result for the definition of standard sequent calculus defined in [14].
} 
Remark 4.6. As it was noted in [14], given condition 4 of the above definition, in any standard Gentzen calculus, the rules $L \wedge$ and $R \vee$ must be the following:

$$
\frac{\Gamma, A, B \Rightarrow \Delta}{\Gamma, A \wedge B \Rightarrow \Delta} L \wedge \quad \frac{\Gamma \Rightarrow \Delta, A, B}{\Gamma \Rightarrow A \vee B, \Delta} R \vee
$$

Remark 4.7. Given a logic $\mathbf{L} \in\left\{\mathbf{K}_{\mathbf{3}}^{\mathbf{w}}, \mathbf{S}_{\mathrm{fde}}, \mathbf{d S}_{\mathrm{fde}}^{w}, \mathbf{S}_{\mathrm{fde}}^{w}\right\}$, any sound standard Gentzen calculus for $\mathbf{L}$ must reject the structural rule of right weakening $(R W)$. In the other case, we would have the following derivation

$$
\frac{\frac{A \Rightarrow A}{A \Rightarrow A, B}}{A \Rightarrow A \vee B} R \vee
$$

where $A$ is a propositional variable. Nonetheless, it is easy to check that $A \nvdash_{\mathbf{L}} A \vee B$.

We want to prove, moreover, that any sound standard Gentzen calculus for any of the logics in question must be incomplete. In order to do that, we need the following lemma:

Lemma 4.8. In any sound standard Gentzen calculus for a logic $\mathbf{L} \in\left\{\mathbf{K}_{\mathbf{3}}^{\mathbf{w}}, \mathbf{S}_{\mathrm{fde}}, \mathbf{d} \mathbf{S}_{\mathrm{fde}}^{w}, \mathbf{S}_{\mathrm{fde}}^{w}\right\}$, the rule for $L \vee$ having as conclusion the sequent $\Gamma, A \vee B \Rightarrow \Delta$, must have two sequents of the following form among its premises: $\Gamma, A \Rightarrow \Delta$ and $\Gamma, B \Rightarrow \Delta$

Proof. Assume that $\Gamma, A \vee B \vDash_{\mathbf{L}} \Delta$ is not the case, so, it is easily provable checking the valuations, that $\Gamma, A \vDash_{\mathbf{L}} \Delta$ or $\Gamma, B \vDash_{\mathbf{L}} \Delta$ must not be the case.

We can, thus, prove the following limitative result

Theorem 4.9. Given a logic $\mathbf{L} \in\left\{\mathbf{K}_{\mathbf{3}}^{\mathbf{w}}, \mathbf{S}_{\mathrm{fde}}, \mathbf{d S}_{\mathrm{fde}}^{w}, \mathbf{S}_{\mathrm{fde}}^{w}\right\}$, it does not admit any standard Gentzen calculus that is sound and complete.

Proof. Let's assume we have a standard Gentzen calculus sound for $\mathbf{L} \in\left\{\mathbf{K}_{\mathbf{3}}^{\mathbf{w}}, \mathbf{S}_{\text {fde }}, \mathbf{d} \mathbf{S}_{\text {fde }}^{w}, \mathbf{S}_{\text {fde }}^{w}\right\}$. If it is also complete, there must be a proof of $A \vee B \Rightarrow A \vee B$ (given that $A \vee B \vDash_{\mathbf{L}} A \vee B$ ). However, the only possible derivation of this sequent must have the following form:

$$
\frac{\frac{A \Rightarrow A, B}{A \Rightarrow A \vee B} R \vee \frac{B \Rightarrow A, B}{B \Rightarrow A \vee B} R \vee \quad P_{j}, \text { with } j=0, \ldots, m}{A \vee B \Rightarrow A \vee B} L \vee
$$

Nevertheless, since right weakening cannot be accepted, there is no way of deriving $A \Rightarrow A, B$. In other words, if the calculus is sound it must be incomplete.

Corollary 4.10. The transparent theories of truth $\mathbf{K}_{3}^{\mathbf{w}} \mathbf{T T}, \mathbf{S}_{\mathrm{fde}} \mathbf{T T}, \mathbf{d} \mathbf{S}_{\mathrm{fde}}^{w} \mathbf{T T}$ and $\mathbf{S}_{\mathrm{fde}}^{w} \mathbf{T T} d o$ not admit any sound and complete standard sequent calculus.

In view of these facts, we will work in the rest of the paper with alternative calculi that we will prove to be sound and complete with respect to the target logics. To this task we now turn.

\section{Sequent calculi for four-valued infectious logics}

\subsection{Philosophical discussion}

The upshot of the previous section is, thus, that there is no standard two-sided sequent calculus for the target infectious logics and the theories of truth built on top of them. The aim of this section is, therefore, to present alternative four-sided sequent calculi for them and to give 
arguments in favor of their adoption. ${ }^{22}$ In doing so, we will argue that these calculi enjoy some of the most important properties that make logicians appreciate standard calculi.

As the following quote by Stefan Wintein ([76]) claims

It is sometimes argued [for instance, ${ }^{23}$ by Ripley [70] and Restall [67]] that the left and right introduction rule of a connective in a two-sided sequent calculus for classical logic specify the meaning of that connective. ([76, p. 36])

Now, as Wintein recalls, various two-sided sequent calculi have been proposed in the literature for $\mathbf{K}_{\mathbf{3}}$ and $\mathbf{L P}$ (see e.g. Avron [5], Arieli and Avron [2] or Beall [10] $]^{24}$ ). This is also the case for $\mathbf{K}_{\mathbf{3}}^{\mathbf{w}}$, PWK (see e.g. [21]), whereas a straightforward application of the techniques to obtain these calculi can be applied to obtain calculi for e.g. $\mathbf{S}_{\text {fde }}$ and $\mathbf{d} \mathbf{S}_{\text {fde }}$.

Thus, we might ask: shouldn't we prefer to characterize our logics by a more standard twosided calculus, over a e.g. four-sided characterization, given the left and right introduction rule of a connective are usually taken to specify the meaning of that connective? To this regard, Wintein's suggestion is that

No we cannot. At least not without much further argument. For not any introduction rule for a connective can be taken to bestow that connective with meaning. How then, do the introduction rules for a connective in a two-sided sequent calculus for classical logic manage to do this; what is so attractive about these rules? Well, the rules (i) have the subformula property, (ii) they introduce exactly one occurrence of a connective in their conclusion and (iii) no other connective is mentioned anywhere else in their formulation. These properties ensure, amongst others, that the meaning of a connective can be understood independently from the meaning of other connectives which, arguably, is an attractive feature from a meaning-theoretic perspective. ([76, p. 36, our emphasis $]$ )

There seem to be a common base for wanting a calculus to exhibit these three trademarks. The basic idea is a very intuitive one: logical constants are synchategorematic terms, i.e. they lack independent meaning. ${ }^{25}$ Their whole contribution to the meaning of a sentence is given by the different inferential ways in which they connect their components. In other words, what may be inferred from a conjunction is not the same as what may be inferred from a disjunction. Also, what is an adequate base for inferring a conjunction is not the same as what is an adequate base for inferring a disjunction. Furthermore, a conjunction, a disjunction, etc. do not provide more information than is provided by the elements that allow to infer the sentence where the

\footnotetext{
${ }^{22}$ We do not take space here to present the corresponding three-sided sequent calculi for $\mathbf{K}_{\mathbf{3}}^{\mathbf{w}} \mathbf{T T}$ and PWKTT for, on the one hand, the calculus for both results from the three-sided sequent calculus for PWK given in [14], plus the following intuitive three-sided rules for the truth predicate

$$
\frac{\Gamma|\Sigma| \Delta, A}{\Gamma,|\Sigma| \Delta, \operatorname{Tr}(\langle A\rangle)} \quad \frac{\Gamma|\Sigma, A| \Delta}{\Gamma,|\Sigma, \operatorname{Tr}(\langle A\rangle)| \Delta} \quad \frac{\Gamma, A|\Sigma| \Delta}{\Gamma, \operatorname{Tr}(\langle A\rangle)|\Sigma| \Delta}
$$

and, on the other hand, the calculus for $\mathbf{K}_{3}^{\mathbf{w}} \mathbf{T T}$ will be the same, although endowed with a different notion of validity, namely, that the three-sided sequent $\Gamma|\Delta| \Delta$ is provable.

${ }^{23}$ But, as remarked by an anonymous referee, the idea is older and was advanced e.g. by Dummet [27], [28], Tennant [73] and Peregrin [53].

${ }^{24} \mathrm{As}$ an anonymous referee kindly points out, the sequent calculi appearing in Beall [10] is there credited in footnote to David Ripley.

${ }^{25}$ This seems a very standard position. As McFarlane says in the following quote taken from [49],

The most venerable approach to demarcating the logical constants identifies them with the language's syncategorematic signs: signs that signify nothing by themselves, but serve to indicate how independently meaningful terms are combined. [49, Section 1]
} 
connective in question is featured. This is why property (i), i.e. the subformula property, seems desirable.

But this does not explain why (ii) and (iii), i.e. that the rules introduce exactly one occurrence of a connective in their conclusion and that no other connective is mentioned anywhere else in their formulation, are desirable properties.

A rationale for having a calculus that enjoys property (ii) might come from the connection between left introduction rules and rejection conditions, on the one hand, and right introduction rules and acceptance conditions, on the other. The idea behind wanting a sequent calculus to satisfy (ii) is that these acceptance and rejection conditions should be given separately, because neither acceptance nor rejection reduces to one another. ${ }^{26}$

Motivation for (iii) can be found in the need for being able to evaluate each candidate for a logical connective independently, and to understand clearly what its meaning is. If the conditions for inferring a connective are the same for the ones for inferring a connective -i.e. both are introduced at the same time, but not necessarily in the same place of a sequent - then it seems not possible to evaluate them separately. Additionally, being able to evaluate them separately is a legitimate goal, because if it is possible to give independent introduction conditions for connective but not for connective $\mathbf{q}$, then there seems to be fewer doubts about $\mathbf{\$}$ 's legitimacy as a logical constant than the ones we have regarding the logicality of $\boldsymbol{\phi}$.

It appears that there are enough reasons to aim at calculi enjoying these properties, however

(...) the rules of none of the two-sided sequent calculi for $\mathbf{L P}$ [Note of the Authors: or for any of the plausible two-sided calculi for $\left.\mathbf{K}_{\mathbf{3}}, \mathbf{K}_{\mathbf{3}}^{\mathbf{w}}, \mathbf{P W K}, \mathbf{S}_{\mathrm{fde}}, \mathbf{d \mathbf { S } _ { \mathrm { fde } }}, \mathbf{S}_{\mathrm{fde}}^{w}, \mathbf{d} \mathbf{S}_{\mathrm{fde}}^{w}\right]$ have these attractive properties. ([76, p. 36, our emphasis])

Hence, it is not clear why a two-sided calculus for any of the logics in question in this paper would be desirable. On the other hand, four-sided sequent calculi seem to be quite suitable, since - as a quick inspection of the rules below will clearly show - the logical part of the calculi we will present in the following section (i.e. the inferences on sequents in which the truth predicate does not appear), do in fact enjoy the above properties: ${ }^{27}$ they enjoy the subformula property, introduce exactly one occurrence of a connective in their conclusion and no other connective is mentioned anywhere else in their formulation. In the following subsection we will take a closer look at them.

\section{2 $\quad$ Four-sided sequent calculi}

In this section we present four-sided calculi that are sound and complete with respect to $\mathbf{S}_{\text {fde }} \mathbf{T T}$, $\mathbf{d S}_{\text {fde }} \mathbf{T T}, \mathbf{S}_{\text {fde }}^{w} \mathbf{T T}$ and $\mathbf{d S}_{\text {fde }}^{w} \mathbf{T T}$, respectively. In order to do this, let us first present the notion of a four-sided sequent and its relation with the valid inferences of these truth theories. Roughly, a four-sided sequent $\Gamma|\Sigma| \Delta \mid \Pi$ is a quadruple of multisets of formulae. Informally, the first two positions correspond to the premises, and the last two to the conclusions.

Definition 5.1. An $\mathcal{M}$-valuation $v$ satisfies a four-sided sequent $\Gamma|\Sigma| \Delta \mid \Pi$ if and only if $v(A) \notin \mathcal{D}$ and $v(\neg A) \in \mathcal{D}$ for some $A \in \Gamma$, or $v(B) \notin \mathcal{D}$ and $v(\neg B) \notin \mathcal{D}$ for some $B \in \Sigma$, or $v(C) \in \mathcal{D}$ and $v(\neg C) \in \mathcal{D}$ for some $C \in \Delta$, or $v(D) \in \mathcal{D}$ and $v(\neg D) \notin \mathcal{D}$ for some $D \in \Pi$. $A$

\footnotetext{
${ }^{26}$ For more about acceptance and rejection conditions as separate and independent attitudes, see [67] and [69].

${ }^{27}$ Of course, it is not the case that the whole calculi, once the truth rules are added, also enjoy the subformula property. But there is nothing special about this. The rules for the truth predicate are the obvious ones. Any sequent calculus that includes them - even the two-sided versions of them-will not exhibit the subformula property, because these rules introduce an atomic formula and may eliminate very complex formulae. Thus, it is not reasonable to demand the subformula property of a sequent calculus for a truth theory. What is desirable is that the base calculus of that theory - i.e. the calculus that results from eliminating the truth rules-has this feature. And all our base calculi below have it.
} 
sequent is valid if and only if it is satisfied by every valuation. A valuation is a counterexample to a sequent if the valuation does not satisfy the sequent. ${ }^{28}$

The satisfaction relation can be, moreover, understood in terms of acceptance and rejection in a given context, as is sometimes asked of standard sequent calculi (cf. mainly [67], and also [70]). In general, when a sentence $A$ is such that $v(A) \in \mathcal{D}$ we will say that it is accepted, and when $v(A) \notin \mathcal{D}$ we will say that it is rejected. We will characterize the above cases in terms of situations where, respectively, the sentence $A$ is rejected and its negation $\neg A$ is accepted, both $A$ and $\neg A$ are rejected, both $A$ and $\neg A$ are accepted, the sentence $A$ is accepted and its negation $\neg A$ is rejected.

Given a theory of truth $\mathbf{L T T} \in\left\{\mathbf{S}_{\text {fde }} \mathbf{T T}, \mathbf{d} \mathbf{S}_{\text {fde }} \mathbf{T T}, \mathbf{S}_{\text {fde }}^{w} \mathbf{T T}, \mathbf{d} \mathbf{S}_{\text {fde }}^{w} \mathbf{T T}\right\}$, due to the previous definitions there is a strong relation between valid inferences and valid four-sided sequents:

\section{$\Gamma \vDash_{\text {LTT }} \Delta \quad$ if and only if $\quad \Gamma|\Gamma| \Delta \mid \Delta$ is valid}

Finally, and in accordance with this, for a given four-sided sequent $\Gamma|\Sigma| \Delta \mid \Pi$ we will label the $\Gamma, \Sigma, \Delta$ and $\Pi$ sides with the corresponding truth-value that is assigned to e.g. $A$ when it is such that, respectively, the sentence $A$ is rejected and its negation $\neg A$ is accepted, both $A$ and $\neg A$ are rejected, both $A$ and $\neg A$ are accepted, the sentence $A$ is accepted and its negation $\neg A$ is rejected. ${ }^{29}$

Moving now to presenting the calculi themselves, the four sequent calculi share their structural rules and the their operational rules for negation and the truth predicate. The differences come with the rules for conjunction and disjunction. The following are the shared rules:

$$
\begin{aligned}
& \overline{A, \Gamma|A, \Sigma| A, \Delta \mid A, \Pi} I d \\
& \frac{\Gamma, A, A|\Sigma| \Delta \mid \Pi}{\Gamma, A|\Sigma| \Delta \mid \Pi} \text { Cont }_{1} \frac{\Gamma|\Sigma, A, A| \Delta \mid \Pi}{\Gamma|\Sigma, A| \Delta \mid \Pi} \text { Cont }_{2} \frac{\Gamma|\Sigma| \Delta, A, A \mid \Pi}{\Gamma|\Sigma| \Delta, A \mid \Pi} \text { Cont }_{3} \frac{\Gamma|\Sigma| \Delta \mid \Pi, A, A}{\Gamma|\Sigma| \Delta \mid \Pi, A} \text { Cont }_{4} \\
& \frac{\Gamma, A|\Sigma| \Delta|\Pi \Gamma| \Sigma, A|\Delta| \Pi}{\Gamma|\Sigma| \Delta \mid \Pi} C_{1} \quad \frac{\Gamma|\Sigma| \Delta, A|\Pi \quad \Gamma| \Sigma, A|\Delta| \Pi}{\Gamma|\Sigma| \Delta \mid \Pi} \text { Cut }_{2} \\
& \frac{\Gamma, A|\Sigma| \Delta|\Pi \quad \Gamma| \Sigma|\Delta, A| \Pi}{\Gamma|\Sigma| \Delta \mid \Pi} C_{3} \quad \frac{\Gamma, A|\Sigma| \Delta|\Pi \quad \Gamma| \Sigma|\Delta| \Pi, A}{\Gamma|\Sigma| \Delta \mid \Pi} \text { Cut }_{4}
\end{aligned}
$$

\footnotetext{
${ }^{28} \mathrm{~A}$ reviewer wonders whether something would be lost if we replace, in Definition 5.1, all occurrences of $\Sigma$ with $\Gamma$, and all occurrences of $\Pi$ with $\Delta$. To this we answer as follows. First, the alternative definition will render the notion of an $\mathcal{M}$-valuation satisfying a four-sided sequent to be undefined for some sequents, i.e. particularly those sequents where $\Gamma \neq \Sigma$ and $\Delta \neq \Pi$. Secondly, this will imply that some sequents of this sort-e.g. $\emptyset|\neg \operatorname{Tr}(\langle\lambda\rangle)| \neg \operatorname{Tr}(\langle\lambda\rangle) \mid \emptyset$ - which are provable in the system, will not be satisfied by any valuation whatsoever. Thirdly, and more importantly, this change will render the rules as unsound. To see this, consider e.g. an application of the rule $\neg 1$ leading from the sequent $A|A| A \mid A$ to $\neg A, A|A| A \mid \emptyset$ - the latter not even complying with the requirements necessary to be satisfied by an $\mathcal{M}$-valuation. For these reasons we believe it is more natural to stick to the above formulation of Definition 5.1, in line with canonical works in $n$-sided calculi such as $[6]$.

${ }^{29}$ This constitutes an inferentialist interpretation of the rules and, in particular, a bilateralist one- following the terminology discussed by Restall and others in e.g. [67] and [70]. But this does not preclude offering a different interpretation for the rules. For instance, as an anonymous referee kindly suggests, a semantic understanding of them is also available. To see this, let us focus, e.g. on the rule $\vee_{2}$ for the logic $\mathbf{S}_{\text {fde }} \mathbf{T T}$. This rule can be read as saying:
}

when neither $A$ nor $B$ are truth-value gaps, and the standard conditions under which disjunction is introduced are met (i.e. either $A$ or $B$ are true), only then we can say that $A \vee B$ is true-thereby introducing a disjunction

with analogous remarks applying to the other rules in this calculus and in the calculi for the remaining systems. 


$$
\begin{aligned}
& \frac{\Gamma|\Sigma, A| \Delta|\Pi \quad \Gamma| \Sigma|\Delta| \Pi, A}{\Gamma|\Sigma| \Delta \mid \Pi} C u_{5} \quad \frac{\Gamma|\Sigma| \Delta, A|\Pi \quad \Gamma| \Sigma|\Delta| \Pi, A}{\Gamma|\Sigma| \Delta \mid \Pi} \mathrm{Cut}_{6} \\
& \frac{\Gamma, A|\Sigma, A| \Delta, A|\Pi \quad \Gamma, A| \Sigma, A|\Delta| \Pi, A \quad \Gamma, A|\Sigma| \Delta, A|\Pi, A \quad \Gamma| \Sigma, A|\Delta, A| \Pi, A}{\Gamma|\Sigma| \Delta \mid \Pi} \text { Derived Cut } \\
& \frac{\Gamma|\Sigma| \Delta \mid \Pi, A}{\Gamma, \neg A|\Sigma| \Delta \mid \Pi} \neg 1 \quad \frac{\Gamma|\Sigma, A| \Delta \mid \Pi}{\Gamma|\Sigma, \neg A| \Delta \mid \Pi} \neg 2 \quad \frac{\Gamma|\Sigma| \Delta, A \mid \Pi}{\Gamma|\Sigma| \Delta, \neg A \mid \Pi} \neg 3 \quad \frac{\Gamma, A|\Sigma| \Delta \mid \Pi}{\Gamma|\Sigma| \Delta \mid \Pi, \neg A} \neg 4 \\
& \frac{\Gamma|\Sigma| \Delta \mid \Pi, A}{\Gamma|\Sigma| \Delta \mid \Pi, \operatorname{Tr}(\langle A\rangle)} \operatorname{Tr}_{1} \frac{\Gamma|\Sigma, A| \Delta \mid \Pi}{\Gamma|\Sigma, \operatorname{Tr}(\langle A\rangle)| \Delta \mid \Pi} \operatorname{Tr}_{2} \frac{\Gamma|\Sigma| \Delta, A \mid \Pi}{\Gamma|\Sigma| \Delta, \operatorname{Tr}(\langle A\rangle) \mid \Pi} \operatorname{Tr}_{3} \frac{\Gamma, A|\Sigma| \Delta \mid \Pi}{\Gamma, \operatorname{Tr}(\langle A\rangle)|\Sigma| \Delta \mid \Pi} \operatorname{Tr}_{4}
\end{aligned}
$$


Operational rules corresponding to the logic $\mathbf{d} \mathbf{S}_{\text {fde }} \mathbf{T T}$ :

$$
\frac{\Gamma, A|\Sigma, A| \Delta|\Pi, A \quad \Gamma, B| \Sigma, B|\Delta| \Pi, B \quad \Gamma, A, B|\Sigma| \Delta \mid \Pi}{\Gamma, A \wedge B|\Sigma| \Delta \mid \Pi} \wedge_{1} \frac{\Gamma|\Sigma| \Delta|\Pi, A \quad \Gamma| \Sigma|\Delta| \Pi, B}{\Gamma|\Sigma| \Delta \mid \Pi, A \wedge B} \wedge_{2} \frac{\Gamma|\Sigma| \Delta, A, B \mid \Pi}{\Gamma|\Sigma| \Delta, A \wedge B \mid \Pi} \wedge_{3}
$$

$\frac{\Gamma|\Sigma, A, B| \Delta|\Pi \quad \Gamma| \Sigma, A|\Delta| \Pi, A \quad \Gamma|\Sigma, B| \Delta \mid \Pi, B}{\Gamma|\Sigma, A \wedge B| \Delta \mid \Pi} \wedge_{4} \frac{\Gamma, A|\Sigma| \Delta|\Pi \quad \Gamma, B| \Sigma|\Delta| \Pi}{\Gamma, A \vee B|\Sigma| \Delta \mid \Pi} \vee_{1} \quad \frac{\Gamma, A|\Sigma, A| \Delta|\Pi, A \quad \Gamma, B| \Sigma, B|\Delta| \Pi, B \quad \Gamma|\Sigma| \Delta \mid \Pi, A, B}{\Gamma|\Sigma| \Delta \mid \Pi, A \vee B} \vee_{2}$

$$
\frac{\Gamma|\Sigma| \Delta, A, B \mid \Pi}{\Gamma|\Sigma| \Delta, A \vee B \mid \Pi} \vee_{3} \quad \frac{\Gamma|\Sigma, A, B| \Delta|\Pi \quad \Gamma, A| \Sigma, A|\Delta| \Pi \quad \Gamma, B|\Sigma, B| \Delta \mid \Pi}{\Gamma|\Sigma, A \vee B| \Delta \mid \Pi} \vee_{4}
$$

Operational rules corresponding to the logic $\mathbf{d S}_{\text {fde }}^{w} \mathbf{T T}$ :

$$
\frac{\Gamma, A|\Sigma| \Delta|\Pi, A \quad \Gamma, B| \Sigma|\Delta| \Pi, B \quad \Gamma, A, B|\Sigma| \Delta \mid \Pi}{\Gamma, A \wedge B|\Sigma| \Delta \mid \Pi} \wedge_{1} \frac{\Gamma|\Sigma| \Delta|\Pi, A \quad \Gamma| \Sigma|\Delta| \Pi, B}{\Gamma|\Sigma| \Delta \mid \Pi, A \wedge B} \wedge_{2} \frac{\Gamma|\Sigma| \Delta, A, B \mid \Pi}{\Gamma|\Sigma| \Delta, A \wedge B \mid \Pi} \wedge_{3}
$$

$\frac{\Gamma|\Sigma, A, B| \Delta|\Pi \quad \Gamma, A| \Sigma, A|\Delta| \Pi, A \quad \Gamma, B|\Sigma, B| \Delta \mid \Pi, B}{\Gamma|\Sigma, A \wedge B| \Delta \mid \Pi} \wedge_{4} \frac{\Gamma, A|\Sigma| \Delta|\Pi \quad \Gamma, B| \Sigma|\Delta| \Pi}{\Gamma, A \vee B|\Sigma| \Delta \mid \Pi} \vee_{1} \quad \frac{\Gamma, A|\Sigma| \Delta|\Pi, A \quad \Gamma, B| \Sigma|\Delta| \Pi, B \quad \Gamma|\Sigma| \Delta \mid \Pi, A, B}{\Gamma|\Sigma| \Delta \mid \Pi, A \vee B} \vee_{2}$

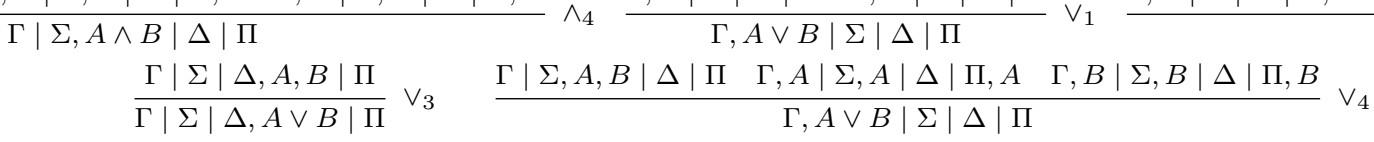

Operational rules corresponding to the logic $\mathbf{S}_{\mathrm{fde}} \mathbf{T T}$ :

$\frac{\Gamma, A|\Sigma| \Delta, A|\Pi, A \quad \Gamma, B| \Sigma|\Delta, B| \Pi, B \quad \Gamma, A, B|\Sigma| \Delta \mid \Pi}{\Gamma, A \wedge B|\Sigma| \Delta \mid \Pi} \wedge_{1} \frac{\Gamma|\Sigma| \Delta|\Pi, A \quad \Gamma| \Sigma|\Delta| \Pi, B}{\Gamma|\Sigma| \Delta \mid \Pi, A \wedge B} \wedge_{2} \frac{\Gamma|\Sigma| \Delta, A, B|\Pi \quad \Gamma| \Sigma|\Delta, A| \Pi, A \quad \Gamma|\Sigma| \Delta, B \mid \Pi, B}{\Gamma|\Sigma| \Delta, A \wedge B \mid \Pi} \wedge_{3}$

$$
\begin{aligned}
& \frac{\Gamma|\Sigma, A, B| \Delta \mid \Pi}{\Gamma|\Sigma, A \wedge B| \Delta \mid \Pi} \wedge_{4} \frac{\Gamma, A|\Sigma| \Delta|\Pi \quad \Gamma, B| \Sigma|\Delta| \Pi}{\Gamma, A \vee B|\Sigma| \Delta \mid \Pi} \vee_{1} \quad \frac{\Gamma, A|\Sigma| \Delta, A|\Pi, A \quad \Gamma, B| \Sigma|\Delta, B| \Pi, B \quad \Gamma|\Sigma| \Delta \mid \Pi, A, B}{\Gamma|\Sigma| \Delta \mid \Pi, A \vee B} \vee_{2} \\
& \frac{\Gamma|\Sigma| \Delta, A, B|\Pi \quad \Gamma, A| \Sigma|\Delta, A| \Pi \quad \Gamma, B|\Sigma| \Delta, B \mid \Pi}{\Gamma|\Sigma| \Delta, A \vee B \mid \Pi} \vee_{3} \frac{\Gamma|\Sigma, A, B| \Delta \mid \Pi}{\Gamma|\Sigma, A \vee B| \Delta \mid \Pi} \vee_{4}
\end{aligned}
$$

Operational rules corresponding to the logic $\mathbf{S}_{\mathrm{fde}}^{w} \mathbf{T T}$ :

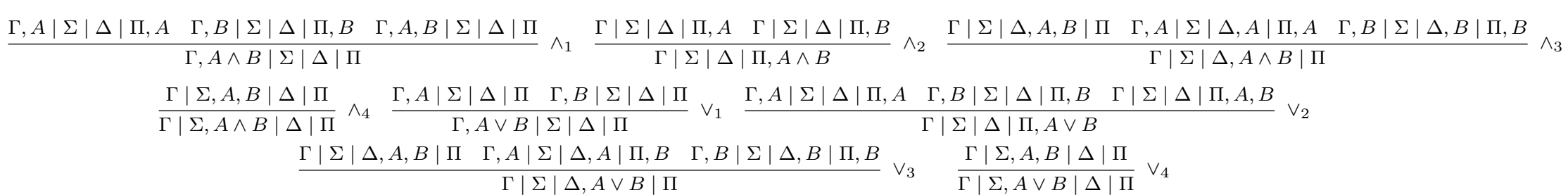


We should highlight - as suggested by an anonymous reviewer - that, for instance, the above rules do not render as valid e.g. inferences of the form: if $\vDash A$, then $\neg A \vDash$, which might not seem to be quite paraconsistent in spirit. Given the equivalence before between valid arguments and valid four-sided sequents, an inference like that would be equivalent to: if $\emptyset|\emptyset| A \mid A$ is a valid sequent, then $\neg A|\neg A| \emptyset \mid \emptyset$ is a valid sequent. But this transition is not allowed, given the previously introduced rules for negation, in any of the four-sided sequent calculi discussed above - as is easy to check by looking at the referred rules.

Moreover, let us remark that in none of the calculi for these theories of truth Cut is eliminable, although it is eliminable in their logical part. In particular, the sequent $\emptyset|\neg \operatorname{Tr}(\langle\lambda\rangle)| \neg \operatorname{Tr}(\langle\lambda\rangle) \mid$ $\emptyset$ can be proved in the calculus, but it cannot be proved without (one of the rules of) Cut. The proof is the following:

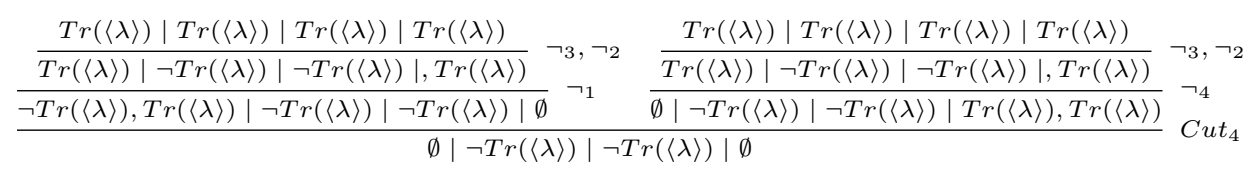

The four proof systems are both sound and complete with regard to their respective consequence relations. As usual, the soundness results are easy to prove. Every axiom is valid, and every rule preserves validity. For the sake of exhaustivity, we will present the completeness result for the four-sided sequent calculus for $\mathbf{d} \mathbf{S}_{\text {fde }} \mathbf{T T}$ next. The completeness proofs for $\mathbf{S}_{\text {fde }} \mathbf{T T}$, $\mathbf{d} \mathbf{S}_{\text {fde }}^{w} \mathbf{T T}$ and $\mathbf{S}_{\text {fde }}^{w} \mathbf{T T}$ are very similar to this one, and are left for the reader as an exercise. ${ }^{30}$

Theorem 5.2 (Completeness of $\mathbf{d S}_{\mathrm{fde}} \mathbf{T T}$ ). For any sequent $\Gamma|\Sigma| \Delta \mid \Pi$, it is valid in $\mathbf{d S}_{\mathrm{fde}} \mathbf{T T}$ only if $\Gamma|\Sigma| \Delta \mid \Pi$ has a proof in the four-sided sequent calculi for $\mathbf{d S}_{\mathrm{fde}} \mathbf{T T}$ previously described.

For the completeness proof of the calculus for $\mathbf{d} \mathbf{S}_{\text {fde }} \mathbf{T T}$ we will use the method of reduction trees, that allows to build, for any given sequent, either a proof of that sequent, or a counterexample to it. ${ }^{31}$ The method also provides a way of building the eventual counterexample to the sequent's validity. As was already specified, this valuation will be a counterexample to the corresponding $\mathbf{d S}_{\text {fde }} \mathbf{T T}$ 's inference.

Definition 5.3. A sequent $S=\Gamma|\Sigma| \Delta \mid \Pi$ is a subsequent of a sequent $S^{\prime}=\Gamma^{\prime}\left|\Sigma^{\prime}\right| \Delta^{\prime} \mid \Pi^{\prime}$ (written $S \sqsubseteq S^{\prime}$ ) if and only if $\Gamma \sqsubseteq \Gamma^{\prime}, \Sigma \sqsubseteq \Sigma^{\prime}, \Delta \sqsubseteq \Delta^{\prime}$ and $\Pi \sqsubseteq \Pi^{\prime}$.

Definition 5.4. A sequent $S=\Gamma|\Sigma| \Delta \mid \Pi$ is the sequent union of a set of sequents $\left[\Gamma_{i}\left|\Sigma_{i}\right| \Delta_{i} \mid \Pi_{i}\right]_{i \in I}$ (written $S=\sqcup\left[\Gamma_{i}\left|\Sigma_{i}\right| \Delta_{i} \mid \Pi_{i}\right]_{i \in I}$ ) iff $\Gamma=\sqcup_{i \in I} \Gamma_{i}, \Sigma=\sqcup_{i \in I} \Sigma_{i}$, $\Delta=\sqcup_{i \in I} \Delta_{i}$ and $\Pi=\sqcup_{i \in I} \Pi_{i}$.

Notice that, in what follows, given a four-sided sequent $\Gamma|\Sigma| \Delta \mid \Pi$ for the case of the theory of truth $\mathbf{d} \mathbf{S}_{\text {fde }} \mathbf{T T}$ we will take the liberty (given Definition 5.1) of saying that a formula is in the f-position, if it belongs to $\Gamma$, that it is in the $\mathbf{i}$-position, if it belongs to $\Sigma$, that it is in the $\mathbf{u}$-position, if it belongs to $\Delta$, and that it is in the $\mathbf{t}$-position, if it belongs to $\Pi$.

\section{Strategy of the proof:}

The construction starts from a root sequent $S_{0}=\Gamma_{0}\left|\Sigma_{0}\right| \Delta_{0} \mid \Pi_{0}$, and then builds a tree in stages, applying at each stage all the operational rules that can be applied, plus Derived Cut "in reverse", i.e. from the conclusion sequent to the premise(s) sequent(s). For the proof, we use

\footnotetext{
${ }^{30} \mathrm{An}$ anonymous referee wonders whether we have considered appealing to a simpler canonical model-like construction to prove completeness. Although we did not consider proving completeness this way, there seem to be no prima facie conceptual problems in applying the usual techniques to do so.

${ }^{31}$ For similar proofs, see e.g. Ripley [69].
} 
an enumeration of the formulae. We will reduce, at each stage, all the formulae in the sequent, starting from the one with the lowest number, then continuing with the formula with the second lowest number, and moving on in this way until the formula with the highest number in the sequent is reduced. ${ }^{32}$

In case a formula appears in more than one side of the sequent, we will start by reducing the formula that appears on the $\mathbf{f}$-position and then proceed to the $\mathbf{i}$-position, the $\mathbf{u}$-position and the t-position, respectively. The final step, at each stage $n$ of the reduction process, will be an application of the Derived Cut rule to the $n$ th-formula in the enumeration. If we apply a multi-premise rule, we will generate more branches that will need to be reduced. If we apply a single-premise rule, we just extend the branch with one more leaf. We will only add formulae at each stage, without erasing any of them.

As a result of the process just described, every branch will be ordered by the subsequent relation. Any branch that has an axiom as it topmost sequent will be closed. A branch that is not closed is considered open. This procedure is repeated until every branch is closed, or until there is an infinite open branch. If every branch is closed, then the resulting tree itself is a proof of the root sequent. If there is an infinite open branch $Y$, we can use it to build a counterexample to the root sequent.

More formally, in stage 0 we will just find the root sequent $S_{0}$. If it is an axiom, the branch is closed. For any stage $n+1$, one of two following things might happen:

1. For all branches in the tree after stage $n$, if the tip is an axiom, the branch is closed.

2. For open branches: For each formula $A$ in a sequent position in each open branch, if $A$ already occurred in that sequent position in that branch (i.e. $A$ has not been generated during stage $n+1$ ), and $A$ has not already been reduced during stage $n+1$, then reduce $A$ as is shown below.

- If $A$ is a negation $\neg B$, then: if $A$ is in the $\mathbf{f}$ (resp. the $\mathbf{i}$ or $\mathbf{u}$ or $\mathbf{t}$ ) position, extend the branch by copying its current tip and adding $B$ to the $\mathbf{t}$ (resp. the $\mathbf{i}$ or $\mathbf{u}$ or $\mathbf{f}$ ) position.

- If $A$ is a conjunction $B \wedge C$, then:

(i) if $A$ is in the $\mathbf{f}$ position, split the branch in two: extend the first by copying its current tip and adding $B$ to the $\mathbf{f}$ position; and extend the second by copying its current tip and adding $C$ to the $\mathbf{f}$ position.

(ii) If $A$ is in the $\mathbf{i}$ position, split the branch in three: extend the first by copying the current tip and adding $B$ to the positions $\mathbf{i}$ and $\mathbf{t}$; extend the second by copying the

\footnotetext{
${ }^{32}$ For the interested readers, we carry out here a derivation of Derived Cut. It has two subderivations, $a$ and $b$. Here is $a$ :

$$
\frac{\Gamma, A|\Sigma, A| \Delta, A|\Pi \quad \Gamma, A| \Sigma, A|\Delta| \Pi, A}{\frac{\Gamma, A|\Sigma, A| \Delta \mid \Pi}{2} C_{t} \frac{\Gamma, A|\Sigma, A| \Delta, A|\Pi \quad \Gamma, A| \Sigma|\Delta, A| \Pi, A}{\Gamma, A|\Sigma| \Delta, A \mid \Pi} \text { Cut }_{2}} \text { Cut }_{5}
$$
}

The following is subderivation $b$ :

$$
\frac{\Gamma, A|\Sigma, A| \Delta|\Pi, A \quad \Gamma, A| \Sigma|\Delta, A| \Pi, A}{\Gamma, A|\Sigma| \Delta \mid \Pi, A} C u t_{2} \frac{\Gamma, A|\Sigma, A| \Delta|\Pi, A \quad \Gamma| \Sigma, A|\Delta, A| \Pi, A}{\Gamma|\Sigma, A| \Delta \mid \Pi, A} \text { Cut }_{1}
$$

Now, finally, we put together the two subderivations, and derive Derived Cut from several applications of Cut.

$$
\frac{\frac{a}{\Gamma, A|\Sigma| \Delta \mid \Pi} \frac{b}{\Gamma|\Sigma| \Delta \mid \Pi, A}}{\Gamma|\Sigma| \Delta \mid \Pi} \mathrm{Cut}_{4}
$$


current tip and adding $C$ to the positions $\mathbf{i}$ and $\mathbf{t}$; and extend the third by copying the current tip and adding both $B$ and $C$ to the $\mathbf{i}$ position.

(iii) If $A$ is in the $\mathbf{u}$ position, extend the branch by copying its current tip and adding both $B$ and $C$ to the $\mathbf{u}$ position.

(iv) If $A$ is in the $\mathbf{t}$ position, split the branch in two: extend the first by copying the current tip and adding $B$ to the $\mathbf{t}$ position; and extend the second by copying the current tip and adding $C$ to the $\mathbf{t}$ position.

- If $A$ is a disjunction $B \vee C$, then:

(i) if $A$ is in the $\mathbf{f}$ position, split the branch in two: extend the first by copying the current tip and adding $B$ to the position $\mathbf{f}$, and extend the second by copying the current tip and adding $C$ to the position $\mathbf{f}$.

(ii) If $A$ is in the n position, split the branch in three: extend the first by copying the current tip and adding $B$ to the positions $\mathbf{f}$ and $\mathbf{i}$; extend the second by copying the current tip and adding $C$ to the positions $\mathbf{f}$ and $\mathbf{i}$; and extend the third by copying the current tip and adding both $B$ and $C$ to the $\mathbf{i}$ position.

(iii) If $A$ is in the $\mathbf{u}$ position, extend the branch by copying its current tip and adding both $B$ and $C$ to the $\mathbf{u}$ position.

(iv) If $A$ is in the $\mathbf{t}$ position, split the branch in three: extend the first by copying the current tip and adding $B$ to the $\mathbf{f}, \mathbf{i}$ and $\mathbf{t}$ positions; extend the second by copying the current tip and adding $C$ to the $\mathbf{f}, \mathbf{i}$ and $\mathbf{t}$ positions; and extend the third by copying the current tip and adding both $B$ and $C$ to the t position.

- If $A$ is a truth assertion $\operatorname{Tr}(\langle B\rangle)$, then if $A$ is in the $\mathbf{f}$ (resp. the $\mathbf{i}$ or $\mathbf{u}$ or $\mathbf{t}$ ) position, extend the branch by copying its current tip and adding $B$ to the $\mathbf{f}$ (resp. the $\mathbf{i}$ or $\mathbf{u}$ or $\mathbf{t}$ ) position.

- If $A$ is a propositional letter, then do nothing.

We will also apply the Derived Cut rule at each step. Consider the $n$th formula in the enumeration of formulae and call it $A$. Now extend each branch using the Derived Cut rule. For each open branch, if its tip is $\Gamma|\Sigma| \Delta \mid \Pi$, split it in four and extend, respectively, the new branches with the sequents

$$
\frac{\Gamma, A|\Sigma, A| \Delta, A \mid \Pi}{\vdots} \quad \frac{\Gamma, A|\Sigma| \Delta, A \mid \Pi, A}{\vdots} \quad \frac{\Gamma, A|\Sigma, A| \Delta \mid \Pi, A}{\vdots} \quad \frac{\Gamma|\Sigma, A| \Delta, A \mid \Pi, A}{\vdots}
$$

Now we need to repeat this procedure until every branch is closed, or, if that does not happen, until there is an infinite open branch. If the first scenario obtains, then the tree itself is a proof of the root sequent, because each step will be the result of an application of a structural or operational rule to the previous steps. If the second scenario obtains, we can use the infinite open branch to build a counterexample.

Lemma 5.5. In infinite open branches, every formula will appear in three of the four sides of the sequents.

Notice that if in fact there is an infinite open branch $Y$, then the Derived Cut rule will have been used infinitely many times. Thus, every formula will appear at some point in the branch in one of the four sides for the first time, and will remain there in every step afterwards. Now, we first collect all sequents of the infinite open branch $Y$ into one single sequent $S_{\omega}=\Gamma_{\omega}\left|\Sigma_{\omega}\right|$ $\Delta_{\omega} \mid \Pi_{\omega}=\sqcup\{S \mid S$ is a sequent of $Y\}$. Notice that, as Derived Cut has been applied infinitely 
many times in the construction of the branch, every formula will occur in exactly three places in $S_{\omega} \cdot{ }^{33}$

Lemma 5.6. For formulas in infinite open branches, there will be a valuation such that no formula in the sequent gets the value associated with the place where it occurs.

There will be a valuation such that no formula in the sequent gets the value associated with the place where it occurs (i.e. $\mathbf{f}$ or $\mathbf{i}$ or $\mathbf{u}$ or $\mathbf{t}$ if the formula occurs in the $\mathbf{f}$ or $\mathbf{i}$ or $\mathbf{u}$ or $\mathbf{t}$ side, respectively). Hence, for each formula $A$ in the sequent, $v$ will give $A$ a value different from the ones corresponding to the sides or positions where $A$ appears in the sequent. But that includes all the formulae in the initial and finite sequent $S_{0}$. That valuation, then, will also be a counterexample to $S_{0}$. Therefore that valuation will be a counterexample to the sequent being considered. This is provided through an induction over the complexity of the formula.

Thus, for atomic formulae $A$ (propositional letters and truth assertions), $v(A)=\mathbf{f}$, or $v(A)=$ $\mathbf{i}$, or $v(A)=\mathbf{u}$, or $v(A)=\mathbf{t}$ iff $A$ does not appear in, respectively, $\Gamma_{\omega}$ or $\Sigma_{\omega}$ or $\Delta_{\omega}$ or $\Pi_{\omega}$.

If $\operatorname{Tr}(\langle B\rangle)$ appears on the $\mathbf{f}$ or $\mathbf{i}$ or $\mathbf{u}$ or $\mathbf{t}$ side of the sequent, the result of reducing it renders is a sequent with an additional $B$ on the $\mathbf{f}$ or $\mathbf{i}$ or $\mathbf{u}$ or $\mathbf{t}$ side, correspondingly. In the cases where $\operatorname{Tr}(\langle B\rangle)$ appears in an infinite open branch, then $v(\operatorname{Tr}(\langle B\rangle))=\mathbf{f}$ or $\mathbf{i}$ or $\mathbf{u}$ or $\mathbf{t}$, respectively, iff $B$ does not appear in $\Gamma_{\omega}, \Sigma_{\omega}, \Delta_{\omega}$ or $\Pi_{\omega}$, respectively. Notice that $B$ will appear in exactly the places where $\operatorname{Tr}(\langle B\rangle)$ appears. As any formula in a sequent that corresponds to an infinite open branch, $B$ appears in exactly three places. If $\operatorname{Tr}(\langle B\rangle))$ appears in the only place where $B$ does not appear, then, as $\operatorname{Tr}(\langle B\rangle)$ will eventually be reduced, $B$ will appear in the only place where it does not appear until that moment in branch. But then that sequent will be an axiom, and thus the branch will be closed. This is the only possibility that we need to consider and, thus, $\operatorname{Tr}(\langle B\rangle)$ cannot appear in less places than $B$ : as any formula in a sequent corresponding to an infinite open branch, it has to appear in exactly three places.

The rules for reducing formulae can be used to show that, if none of the components of complex formulae receives the truth-value associated with the place in which they appear in $S_{\omega}$, neither will the compound. We will not see, due to limitations of space, how this method works in detail for negations and disjunctions but, nevertheless, we will examine with some detail now how to reduce conjunctions, noting that for disjunctions and negations we should proceed in a similar fashion.

Let us now focus, then, on conjunctions. We need to consider four possible cases:

- (i) $B \wedge C$ appears in the positions $\mathbf{f}, \mathbf{i}$ and $\mathbf{u}$, or

- (ii) $B \wedge C$ appears in positions $\mathbf{f}$, i and $\mathbf{t}$, or

- (iii) $B \wedge C$ appears in positions $\mathbf{f}, \mathbf{u}$ and $\mathbf{t}$, or

- (iv) $B \wedge C$ appears in positions $\mathbf{i}, \mathbf{u}$ and $\mathbf{t}$.

CASE (i): at some stage $n$, the conjunction will appear from the first time in the three positions of the sequent, $\mathbf{f}, \mathbf{i}$ and $\mathbf{u}$. There will have been a first stage where the three of them appear. So let's focus on the sequent: $\Gamma, B \wedge C|\Sigma, B \wedge C| \Delta, B \wedge C \mid \Pi$.

In the next step, the three appearances of the conjunction in the positions $\mathbf{f}, \mathbf{i}$ and $\mathbf{u}$ will be reduced. The reduction of the conjunction on the $\mathbf{f}$-side will generate three new sequents:

- (i.a) $\Gamma, B \wedge C, B, C|\Sigma, B \wedge C| \Delta, B \wedge C \mid \Pi$,

- (i.b) $\Gamma, B \wedge C, B|\Sigma, B \wedge C, B| \Delta, B \wedge C \mid \Pi, B$ and

- (i.c) $\Gamma, B \wedge C, C|\Sigma, B \wedge C, C| \Delta, B \wedge C \mid \Pi, C$.

\footnotetext{
${ }^{33}$ It cannot occur in the four places, because then there will be some finite stage $n$ where the formula appears for the first time in the branch in the four sides. But then that sequent will be an axiom, and therefore the branch will be closed.
} 
In case (i.a), the result of reducing the conjunction $B \wedge C$ on the positions $\mathbf{i}$ and $\mathbf{u}$ will generate three new sequents:

- (i.a.1) $\Gamma, B \wedge C, B, C|\Sigma, B \wedge C, B, C| \Delta, B \wedge C, B, C \mid \Pi$,

- (i.a.2) $\Gamma, B \wedge C, B, C|\Sigma, B \wedge C, B| \Delta, B \wedge C, B, C \mid \Pi, B$ and

- (i.a.3) $\Gamma, B \wedge C, B, C|\Sigma, B \wedge C, C| \Delta, B \wedge C, B, C \mid \Pi, C$.

Cases (i.a.2) and (1a3) are axioms. In (i.a.2), $B$ appears in the four positions. And in (i.a.3), $C$ appears in the four positions. Thus, those branches will be closed. The only possibly open branch will correspond to (i.a.1), in which case both $B$ and $C$ appear in positions $\mathbf{f}$, i and $\mathbf{u}$. Thus, $v(B)=v(C)=\mathbf{t}$. And thus, by the compositional behaviour of the conjunction, $v(B \wedge C)=\mathbf{t}$, i.e. the value that correspond to the only position where the conjunction does not appear in the original sequent.

In case (i.b), the result of reducing the conjunction $B \wedge C$ on the positions $\mathbf{i}$ and $\mathbf{u}$ will generate three new sequents:

- (i.b.1) $\Gamma, B \wedge C, B|\Sigma, B \wedge C, B, C| \Delta, B \wedge C, B, C \mid \Pi, B$,

- (i.b.2) $\Gamma, B \wedge C, B|\Sigma, B \wedge C, B| \Delta, B \wedge C, B, C \mid \Pi, B$ and

- (i.b.3) $\Gamma, B \wedge C, B|\Sigma, B \wedge C, B, C| \Delta, B \wedge C, B, C \mid \Pi, B, C$.

Cases (i.b.1), (i.b.2) and (i.b.3) are axioms, because $B$ will appear on the four positions on the sequent. Thus, none of these branches can be part of the infinite open branch.

In case (i.c), the result of reducing the conjunction $B \wedge C$ on the positions $\mathbf{i}$ and $\mathbf{u}$ will generate three new sequents:

- (i.b.1) $\Gamma, B \wedge C, C|\Sigma, B \wedge C, B, C| \Delta, B \wedge C, B, C \mid \Pi, C$,

- (i.b.2) $\Gamma, B \wedge C, C|\Sigma, B \wedge C, B, C| \Delta, B \wedge C, B, C \mid \Pi, B, C$ and

- (i.b.3) $\Gamma, B \wedge C, C|\Sigma, B \wedge C, C| \Delta, B \wedge C, B, C \mid \Pi, C$.

Cases (i.c.1), (i.c.2) and (i.c.3) are axioms, because $C$ will appear on the four positions on the sequent. Thus, none of these branches can be part of the infinite open branch.

CASE (ii): at some stage $n, B \wedge C$ will appear from the first time in the three positions of the sequent, $\mathbf{f}, \mathbf{i}$ and $\mathbf{t}$. Thus, there will have been a first stage where the three of them appear. So let's focus on the sequent $\Gamma, B \wedge C|\Sigma, B \wedge C| \Delta \mid \Pi, B \wedge C$.

In the next step, the three appearances of $B \wedge C$ in the positions $\mathbf{f}, \mathbf{i}$ and $\mathbf{t}$ will be reduced. The reduction of the conjunction on the $\mathbf{f}$ position will generate three new sequents:

- (ii.a) $\Gamma, B \wedge C, B, C|\Sigma, B \wedge C| \Delta \mid \Pi, B \wedge C$,

- (ii.b) $\Gamma, B \wedge C, B|\Sigma, B \wedge C, B| \Delta \mid \Pi, B \wedge C, B$ and

- (ii.c) $\Gamma, B \wedge C, C|\Sigma, B \wedge C, C| \Delta \mid \Pi, B \wedge C, C$.

The reduction of the conjunction on the position $\mathbf{i}$ will generate, in each case, three new sequents:

- (ii.a.1) $\Gamma, B \wedge C, B, C|\Sigma, B \wedge C, B, C| \Delta \mid \Pi, B \wedge C$,

- (ii.a.2) $\Gamma, B \wedge C, B, C|\Sigma, B \wedge C, B| \Delta \mid \Pi, B \wedge C, B$,

- (ii.a.3) $\Gamma, B \wedge C, B, C|\Sigma, B \wedge C, C| \Delta \mid \Pi, B \wedge C, C$.

- (ii.b.1) $\Gamma, B \wedge C, B|\Sigma, B \wedge C, B, C| \Delta \mid \Pi, B \wedge C, B$,

- (ii.b.2) $\Gamma, B \wedge C, B|\Sigma, B \wedge C, B| \Delta \mid \Pi, B \wedge C, B$,

- (ii.b.3) $\Gamma, B \wedge C, B|\Sigma, B \wedge C, B, C| \Delta \mid \Pi, B \wedge C, B, C$.

- (ii.c.1) $\Gamma, B \wedge C, C|\Sigma, B \wedge C, B, C| \Delta \mid \Pi, B \wedge C, C$,

- (ii.c.2) $\Gamma, B \wedge C, B, C|\Sigma, B \wedge C, B, C| \Delta \mid \Pi, B \wedge C, B, C$,

- (ii.c.3) $\Gamma, B \wedge C, C|\Sigma, B \wedge C, C| \Delta \mid \Pi, B \wedge C, C$. 
Finally, the reduction of the conjunction in the position $\mathbf{t}$ will generate, in each subcase, two new sequents, that are the following:

- (ii.a.1.1) $\Gamma, B \wedge C, B, C|\Sigma, B \wedge C, B, C| \Delta \mid \Pi, B \wedge C, B$,

- (ii.a.1.2) $\Gamma, B \wedge C, B, C|\Sigma, B \wedge C, B, C| \Delta \mid \Pi, B \wedge C, C$,

- (ii.a.2.1) $\Gamma, B \wedge C, B, C|\Sigma, B \wedge C, B| \Delta \mid \Pi, B \wedge C, B$,

- (ii.a.2.2) $\Gamma, B \wedge C, B, C|\Sigma, B \wedge C, B| \Delta \mid \Pi, B \wedge C, B, C$

- (ii.a.3.1) $\Gamma, B \wedge C, B, C|\Sigma, B \wedge C, B, C| \Delta \mid \Pi, B \wedge C, B, C$,

- (ii.a.3.2) $\Gamma, B \wedge C, B, C|\Sigma, B \wedge C, C| \Delta \mid \Pi, B \wedge C, C$.

In cases (ii.a.1.1), (ii.a.2.1) and (ii.a.2.2), the only place where $C$ does not appear is in the $\mathbf{u}$ position. Thus, $v(B)=\mathbf{u}$, and thus, $v(B \wedge C)=\mathbf{u}$, since $\mathbf{u}$ is infectious in this logic. In cases (ii.a.1.2), (ii.a.3.1) and (ii.a.3.2), the only place where $C$ does not appear is in $\mathbf{u}$. Thus, $v(C)=\mathbf{u}$, and thus, $v(B \wedge C)=\mathbf{u}$ for the same reasons.

CAse (iii): at some stage $n, B \wedge C$ will appear from the first time in the three positions of the sequent $\mathbf{f}, \mathbf{u}$ and $\mathbf{t}$. Thus, there will have been a first stage where the three of them appear. So let's focus on the sequent $\Gamma, B \wedge C|\Sigma| \Delta, B \wedge C \mid \Pi, B \wedge C$.

In the next stage, the reduction of the conjunction on the f-side will generate three new sequents:

- (iii.a) $\Gamma, B \wedge C, B, C|\Sigma| \Delta, B \wedge C \mid \Pi, B \wedge C$,

- (iii.b) $\Gamma, B \wedge C, B|\Sigma, B| \Delta, B \wedge C \mid \Pi, B \wedge C, B$ and

- (iii.c) $\Gamma, B \wedge C, C|\Sigma, C| \Delta, B \wedge C \mid \Pi, B \wedge C, C$.

In (iii.a), the result of reducing the conjunction $B \wedge C$ on the u-position will generate, in each case, one new sequent. The three new sequents will then be:

- (iii.a.1) $\Gamma, B \wedge C, B, C|\Sigma| \Delta, B \wedge C, B, C \mid \Pi, B \wedge C$,

- (iii.b.1) $\Gamma, B \wedge C, B|\Sigma, B| \Delta, B \wedge C, B, C \mid \Pi, B \wedge C, B$ and

- (iii.c.1) $\Gamma, B \wedge C, C|\Sigma, C| \Delta, B \wedge C, B, C \mid \Pi, B \wedge C, C$.

In each case, the result of reducing the conjunction on the $\mathbf{t}$-position will generate two new sequent, one with $B$ on the t-position, the other with $C$ on the t-position:

- (iii.a.1.1) $\Gamma, B \wedge C, B, C|\Sigma| \Delta, B \wedge C, B, C \mid \Pi, B \wedge C, B$,

- (iii.a.1.2) $\Gamma, B \wedge C, B, C|\Sigma| \Delta, B \wedge C, B, C \mid \Pi, B \wedge C, C$,

- (iii.b.1.1) $\Gamma, B \wedge C, B|\Sigma, B| \Delta, B \wedge C, B, C \mid \Pi, B \wedge C, B$,

- (iii.b.1.2) $\Gamma, B \wedge C, B|\Sigma, B| \Delta, B \wedge C, B, C \mid \Pi, B \wedge C, B, C$,

- (iii.c.1.1) $\Gamma, B \wedge C, C|\Sigma, C| \Delta, B \wedge C, B, C \mid \Pi, B \wedge C, B, C$ and

- (iii.c.1.2) $\Gamma, B \wedge C, C|\Sigma, C| \Delta, B \wedge C, B, C \mid \Pi, B \wedge C, C$

In cases (iii.b.1.1) and (iii.b.1.2), $B$ will appear on the four positions, and thus the sequent will be an axiom, and the branch will be closed. A similar thing happens with cases (iii.c.1.1) and (iii.c.1.2), where $C$ appears on the four positions, and thus the sequent is an axiom, and the branch will be closed. In (iii.a.1.1), $B$ will appear on the $\mathbf{f}, \mathbf{u}$ and $\mathbf{t}$ position. Thus, $v(B)=\mathbf{i}$. $C$, on the other hand, appears on the $\mathbf{f}$ and $\mathbf{u}$ sides. Thus, either $v(C)=\mathbf{i}$ or $v(C)=\mathbf{t}$. In either case, $v(B \wedge C)=\mathbf{i}$. In case (iii.a.1.2), $C$ will appear on the $\mathbf{f}, \mathbf{u}$ and $\mathbf{t}$ position. Thus, $v(C)=\mathbf{i}$. $C$, on the other hand, appears on the $\mathbf{f}$ and $\mathbf{u}$ sides. Thus, either $v(B)=\mathbf{i}$ or $v(B)=\mathbf{t}$. And in either case, $v(B \wedge C)=\mathbf{i}$.

CASE (iv): at some stage $n, B \wedge C$ will appear from the first time in the three positions of the sequent $\mathbf{i}, \mathbf{u}$ and $\mathbf{t}$. So let's focus on the sequent $\Gamma,|\Sigma, B \wedge C| \Delta, B \wedge C \mid \Pi, B \wedge C$.

The reduction of the conjunction on the position $\mathbf{i}$ will generate, in each case, three new sequents:

- (iv.a) $\Gamma|\Sigma, B \wedge C, B, C| \Delta, B \wedge C \mid \Pi, B \wedge C$, 
- (iv.b) $\Gamma|\Sigma, B \wedge C, B| \Delta, B \wedge C \mid \Pi, B \wedge C, B$ and

- (iv.c) $\Gamma|\Sigma, B \wedge C, C| \Delta, B \wedge C \mid \Pi, B \wedge C, C$.

The result of reducing the conjunction on the position $\mathbf{u}$ will just add, in each case, both $B$ and $C$ to the $\mathbf{u}$-position. This are the three new sequents that will be obtained:

- (iv.a.1) $\Gamma|\Sigma, B \wedge C, B, C| \Delta, B \wedge C, B, C \mid \Pi, B \wedge C$,

- (iv.b.1) $\Gamma|\Sigma, B \wedge C, B| \Delta, B \wedge C, B, C \mid \Pi, B \wedge C, B$ and

- (iv.c.1) $\Gamma|\Sigma, B \wedge C, C| \Delta, B \wedge C, B, C \mid \Pi, B \wedge C, C$.

Finally, the reduction of the conjunction on the t-position will result, in each case, in two new sequents:

- (iv.a.1.1) $\Gamma|\Sigma, B \wedge C, B, C| \Delta, B \wedge C, B, C \mid \Pi, B \wedge C, B$.

- (iv.a.1.2) $\Gamma|\Sigma, B \wedge C, B, C| \Delta, B \wedge C, B, C \mid \Pi, B \wedge C, C$.

- (iv.b.1.1) $\Gamma|\Sigma, B \wedge C, B| \Delta, B \wedge C, B, C \mid \Pi, B \wedge C, B$.

- (iv.b.1.2) $\Gamma|\Sigma, B \wedge C, B| \Delta, B \wedge C, B, C \mid \Pi, B \wedge C, B, C$.

- (iv.c.1.1) $\Gamma|\Sigma, B \wedge C, C| \Delta, B \wedge C, B, C \mid \Pi, B \wedge C, B, C$.

- (iv.c.1.2) $\Gamma|\Sigma, B \wedge C, C| \Delta, B \wedge C, B, C \mid \Pi, B \wedge C, C$.

In (iv.a.1.1), the only position where $B$ does not appear is the $\mathbf{f}$ position. Thus, $v(B)=\mathbf{f}$. $C$ appears on the $\mathbf{i}$ and $\mathbf{u}$ positions. Thus, either $v(C)=\mathbf{t}$ or $\mathbf{f}$. Thus, $v(B \wedge C)=\mathbf{f}$. In (iv.a.1.2), the only position where $C$ does not appear is the $\mathbf{f}$ position. Thus, $v(C)=\mathbf{f}$. $B$ appears in the $\mathbf{i}$ and $\mathbf{u}$ positions. Thus, either $v(B)=\mathbf{t}$ or $\mathbf{f}$. Hence, $v(B \wedge C)=\mathbf{f}$. In (iv.b.1.1), the only position where $B$ does not appear is the $\mathbf{f}$ position. Thus, $v(B)=\mathbf{f}$. $C$ appears on the $\mathbf{u}$ position. Thus, either $v(C)=\mathbf{f}$, or $\mathbf{i}$, or t. Hence, $v(B \wedge C)=\mathbf{f}$. In (iv.b.1.2), the only position where $B$ does not appear is the f position. Thus, $v(B)=\mathbf{f} . C$ appears on positions $\mathbf{u}$ and $\mathbf{t}$. Thus, either $v(C)=\mathbf{f}$, or i. Hence, $v(B \wedge C)=\mathbf{f}$. In (iv.c.1.1), the only position where $C$ does not appear is the $\mathbf{f}$ position. Thus, $v(C)=\mathbf{f}$. $B$ appears on the $\mathbf{i}$ and $\mathbf{u}$ positions. Thus, either $v(B)=\mathbf{f}$, or $\mathbf{i}$. Whence, $v(B \wedge C)=\mathbf{f}$. In (iv.c.1.2), the only position where $C$ does not appear is the $\mathbf{f}$ position. Thus, $v(C)=\mathbf{f}$. $B$ appears on the $\mathbf{i}$ and $\mathbf{u}$ positions. Thus, either $v(B)=\mathbf{f}$, or $\mathbf{i}$, or $\mathbf{t}$. Whence, finally, $v(B \wedge C)=\mathbf{f}$.

By completing the induction along these lines, we can show that we can construct a valuation such that no formula receives the value associated with any place where it appears in $S_{\omega}$. But, as we know, that includes all the formulae in the initial and finite sequent $S_{0}$. That valuation, then, will also be a counterexample to $S_{0}$, which is what we were looking for. Thus, for any sequent $S$, either it has a proof or it has a counterexample.

\section{Coda: functionally complete extensions}

In this section we will consider some functionally complete extensions of the infectious logics presented in this paper, by means of the addition of Post-like cyclic operators (cf. [54] and [55]). After doing that we will discuss the problematic upshot of having theories of truth so powerful that they will be prone to revenge paradoxes. To escape this problem we will mention the possibility of embracing the so-called "Goodship Project" (cf. [39]), thanks to which revenge paradoxes are avoided by means of expressing self-referential sentences through equivalences and not through identities. It is worth noting, first, that throughout this paper we express self-reference through identities - as usual - and, secondly, that since expressing self-reference via equivalences with the help of a non-classical conditional is not standard, we just mention this alternative in this Coda, leaving a deep discussion about this point for further research. 
Let us, then, consider why should we bother to discuss functionally complete extensions for the previously presented infectious logics and, therefore, to present functionally complete extensions for the previously defined transparent theories of truth. The answer is that transparent theories of truth are not only usually proposed as a non-hierarchical and positive answer to the Tarskian claim that formal theories cannot represent their own notion of truth, but also as a means to the end of having semantically closed theories. By that we mean theories that are able, contra Tarski's limitative results, to non-trivially represent every semantic concept-and, therefore, every semantic concept applicable to the theory itself.

Truth is only one of many notions that would be required to be expressible, if we were to aim at a semantically closed theory. Now, if we were indeed to aim at such powerful theories, a fortiori every extensional notion - that is, every notion expressible through a truth-tableshould be expressible within theories of that sort. This feature is, indeed, closely related to the property of being functionally complete and, thus, a necessary condition for a theory to be semantically closed is for that theory to be functionally complete. This explains why scholars working on transparent theories of truth might be interested in transparent theories of truth built using functionally complete logics.

Now, in order to get functionally complete extensions of the aforementioned four-valued infectious logics, we will follow Post's technique to obtain functionally complete $n$-valued logics, as presented by Malinowski in $[47,547-548] .{ }^{34}$ For the special case of four-valued logics counting with the values $\mathbf{v}_{1}, \mathbf{v}_{2}, \mathbf{v}_{3}$ and $\mathbf{v}_{4}$, Post's requirement to have a sufficient set of connectives that is functionally complete is to have a unary connective $\circlearrowleft$ and a binary connective $\sharp$ that have the corresponding truth-functions

\begin{tabular}{|c|c|c|c|c|c|c|}
\hline & $\circlearrowleft$ & $\sharp$ & $\mathbf{v}_{1}$ & $\mathbf{v}_{2}$ & $\mathbf{v}_{3}$ & $\mathbf{v}_{4}$ \\
\hline $\mathbf{v}_{1}$ & $\mathbf{v}_{2}$ & $\mathbf{v}_{1}$ & $\mathbf{v}_{1}$ & $\mathbf{v}_{2}$ & $\mathbf{v}_{3}$ & $\mathbf{v}_{4}$ \\
\hline $\mathbf{v}_{2}$ & $\mathbf{v}_{3}$ & $\mathbf{v}_{2}$ & $\mathbf{v}_{2}$ & $\mathbf{v}_{2}$ & $\mathbf{v}_{3}$ & $\mathbf{v}_{4}$ \\
\hline $\mathbf{v}_{3}$ & $\mathbf{v}_{4}$ & $\mathbf{v}_{3}$ & $\mathbf{v}_{3}$ & $\mathbf{v}_{3}$ & $\mathbf{v}_{3}$ & $\mathbf{v}_{4}$ \\
\hline $\mathbf{v}_{4}$ & $\mathbf{v}_{1}$ & $\mathbf{v}_{4}$ & $\mathbf{v}_{4}$ & $\mathbf{v}_{4}$ & $\mathbf{v}_{4}$ & $\mathbf{V}_{4}$ \\
\hline
\end{tabular}

In our cases it is easy to observe that none of the aforementioned logics has a unary connective that behaves in that way, but that they indeed have such a binary connective; namely, for each of $\mathbf{S}_{\mathrm{fde}}, \mathbf{d} \mathbf{S}_{\mathrm{fde}}, \mathbf{d} \mathbf{S}_{\mathrm{fde}}^{w}$ and $\mathbf{S}_{\mathrm{fde}}^{w}$, their respective disjunction behaves just like $\sharp$. It is interesting to notice, though, that the mapping in the case of $\mathbf{S}_{\mathbf{f d e}}$ and $\mathbf{d} \mathbf{S}_{\mathbf{f d e}}$ is different than the mapping in the case of $\mathbf{S}_{\text {fde }}^{w}$ and $\mathbf{d} \mathbf{S}_{\text {fde }}^{w}$. For, while in the former case we have $\mathbf{v}_{1} \mapsto \mathbf{f}, \mathbf{v}_{2} \mapsto \mathbf{i}, \mathbf{v}_{3} \mapsto \mathbf{t}$, $\mathbf{v}_{4} \mapsto \mathbf{u}$, in the latter we have $\mathbf{v}_{1} \mapsto \mathbf{f}, \mathbf{v}_{2} \mapsto \mathbf{t}, \mathbf{v}_{3} \mapsto \mathbf{u}_{1}, \mathbf{v}_{4} \mapsto \mathbf{u}_{2}$. This brings our attention to the fact that, if we want to move from these logics to functionally complete extensions thereof, we need to provide each of these pairs of logics with a different connective. Thus, while in the case of $\mathbf{S}_{\text {fde }}$ and $\mathbf{d} \mathbf{S}_{\text {fde }}$ we need $\circlearrowleft_{1}$, in the case of $\mathbf{S}_{\text {fde }}^{w}$ and $\mathbf{d} \mathbf{S}_{\text {fde }}^{w}$ we need $\circlearrowleft_{2}$.

\begin{tabular}{c|ccc|c} 
& $\circlearrowleft^{1}$ & & $\circlearrowleft^{2}$ \\
\hline $\mathbf{f}$ & $\mathbf{i}$ & & $\mathbf{f}$ & $\mathbf{t}$ \\
$\mathbf{i}$ & $\mathbf{t}$ & & $\mathbf{t}$ & $\mathbf{u}_{1}$ \\
$\mathbf{t}$ & $\mathbf{u}$ & & $\mathbf{u}_{1}$ & $\mathbf{u}_{2}$ \\
$\mathbf{u}$ & $\mathbf{f}$ & & $\mathbf{u}_{2}$ & $\mathbf{f}$
\end{tabular}

Given this, let us define the system $\mathbf{S}_{\text {fde }}^{*}$ and $\mathbf{d} \mathbf{S}_{\text {fde }}^{*}$ as the extensions of the logics $\mathbf{S}_{\text {fde }}$ and $\mathbf{d} \mathbf{S}_{\text {fde }}$, respectively, with the connective $\circlearrowleft^{1}$. Analogously, let us define the system $\mathbf{S}_{\text {fde }}^{w}{ }^{*}$ and $\mathbf{d} \mathbf{S}_{\text {fde }}^{w}{ }^{*}$ as the extensions of the logics $\mathbf{S}_{\text {fde }}^{w}$ and $\mathbf{d} \mathbf{S}_{\text {fde }}^{w}$, respectively, with the new connective $\circlearrowleft^{2}$. Each of these functionally complete extensions can receive appropriate four-sided sequent calculi by adding the following four rules, respectively:

\footnotetext{
${ }^{34}$ We will make some notational adjustments that will not affect the result. Notice, additionally, that as is proved in [75], there are no minimal functionally complete sets of functions for algebras with more than three elements.
} 
- Rules for $\mathbf{S}_{\text {fde }}^{*}$ :

$$
\frac{\Gamma|\Sigma| \Delta \mid \Pi, A}{\Gamma\left|\Sigma, \circlearrowleft^{1} A\right| \Delta \mid \Pi} \circlearrowleft_{1}^{1} \quad \frac{\Gamma|\Sigma, A| \Delta \mid \Pi}{\Gamma, \circlearrowleft^{1} A|\Sigma| \Delta \mid \Pi} \circlearrowleft_{2}^{1} \quad \frac{\Gamma|\Sigma| \Delta, A \mid \Pi}{\Gamma|\Sigma| \Delta \mid \Pi, \circlearrowleft^{1} A} \circlearrowleft_{3}^{1} \quad \frac{\Gamma, A|\Sigma| \Delta \mid \Pi}{\Gamma|\Sigma| \Delta, \circlearrowleft^{1} A \mid \Pi} \circlearrowleft_{4}^{1}
$$

- Rules for $\mathbf{d S}_{\text {fde }}^{*}$ :

$$
\frac{\Gamma|\Sigma| \Delta \mid \Pi, A}{\Gamma|\Sigma| \Delta, \circlearrowleft^{1} A \mid \Pi} \circlearrowleft_{1}^{1} \quad \frac{\Gamma|\Sigma, A| \Delta \mid \Pi}{\Gamma|\Sigma| \Delta \mid \Pi, \circlearrowleft^{1} A} \circlearrowleft_{2}^{1} \quad \frac{\Gamma|\Sigma| \Delta, A \mid \Pi}{\Gamma, \circlearrowleft^{1} A|\Sigma| \Delta \mid \Pi} \circlearrowleft_{3}^{1} \quad \frac{\Gamma, A|\Sigma| \Delta \mid \Pi}{\Gamma\left|\Sigma, \circlearrowleft^{1} A\right| \Delta \mid \Pi} \circlearrowleft_{4}^{1}
$$

- Rules for $\mathbf{S}_{\mathrm{fde}}^{w}{ }^{*}$ :

$$
\frac{\Gamma|\Sigma| \Delta \mid \Pi, A}{\Gamma|\Sigma| \Delta, \circlearrowleft^{2} A \mid \Pi} \circlearrowleft_{1}^{2} \quad \frac{\Gamma|\Sigma, A| \Delta \mid \Pi}{\Gamma, \circlearrowleft^{2} A|\Sigma| \Delta \mid \Pi} \circlearrowleft_{2}^{2} \quad \frac{\Gamma|\Sigma| \Delta, A \mid \Pi}{\Gamma\left|\Sigma, \circlearrowleft^{2} A\right| \Delta \mid \Pi} \circlearrowleft_{3}^{2} \quad \frac{\Gamma, A|\Sigma| \Delta \mid \Pi}{\Gamma|\Sigma| \Delta \mid \Pi, \circlearrowleft^{2} A} \circlearrowleft_{4}^{2}
$$

- Rules for $\mathbf{d S}_{\text {fde }}^{w}$ :

$$
\frac{\Gamma|\Sigma| \Delta \mid \Pi, A}{\Gamma\left|\Sigma, \circlearrowleft^{2} A\right| \Delta \mid \Pi} \circlearrowleft_{1}^{2} \quad \frac{\Gamma|\Sigma, A| \Delta \mid \Pi}{\Gamma|\Sigma| \Delta, \circlearrowleft^{2} A \mid \Pi} \circlearrowleft_{2}^{2} \quad \frac{\Gamma|\Sigma| \Delta, A \mid \Pi}{\Gamma, \circlearrowleft^{2} A|\Sigma| \Delta \mid \Pi} \circlearrowleft_{3}^{2} \quad \frac{\Gamma, A|\Sigma| \Delta \mid \Pi}{\Gamma|\Sigma| \Delta \mid \Pi, \circlearrowleft^{2} A} \circlearrowleft_{4}^{2}
$$

Going back now to our main aim of having transparent theories of truth, it is reasonable to ask now how good are these functionally complete extensions as such, i.e. as theories of truth? The answer requires some subtleties: if self-referential sentences are expressed through identities, then the resulting systems will be trivial and, thus, of no use at all. This is easy to observe, since given the logics are functionally complete, a classical negation will be definable such that a Strengthened Liar can be easily formulated in the theory, i.e. a sentence $L$ identical to the sentence ' $L$ is not true'. That there is no stable truth-value assignment for such a sentence is routine to prove, thereby establishing that the extensions we have in mind are trivial.

Thus, the question arises whether or not there is some move that can save functionally complete transparent theories of truth - and, in particular, the extensions of the four-valued infectious logics that we are dealing with - from triviality. To this we answer positively: all that needs to be modified is the way self-reference is expressed. While sometimes it is taken for granted that it is irrelevant whether to express self-referential sentences through identities or equivalences, we affirm that expressing self-referential sentences via equivalences is essential for keeping functionally complete theories of truth away from triviality.

Already in a very fundamental paper (cf. [39]), Laura Goodship suggests that for a transparent theory of truth (or any other naive theory, such as naive set-theory) to remain non-trivial all that needs to be done is to change the way self-reference is achieved. More precisely, by expressing it via a biconditional, defined as the conjunction of two conditionals such that the featured conditional either (1) invalidates Modus Ponens, or (2) invalidates Contraction and Pseudo-Modus Ponens. Therefore, walking down any of these paths will lead to a successful rescue of the functionally complete extensions of the transparent theories of truth built on top of four-valued infectious logics.

Let us notice, furthermore, that Goodship's suggestion of making use of a conditional that invalidates Modus Ponens to express self-reference has been recently followed in the literature by a number of papers. Hitoshi Omori's paper [25] shows how this technique can be applied to render a non-trivial and highly expressive naive set-theory based on the three-valued logic LP. Barrio, Pailos and Szmuc's paper [9] show how to apply this technique to build a functionally complete transparent theory of truth based on a paraconsistent logic closely related to Coniglio and Silvestrini's MPT from [22]. In this vein, Graham Priest's paper [64] also presents a discussion of Goodship's suggestions in connection with his favored position toward semantic paradoxes.

It might be asked (as an anonymous reviewer did) how is it that the functionally complete theories of truth explored above could actually be used-proof theoretically speaking-given they invalidate Modus Ponens. To this we answer that, in functionally complete systems like 
the above, it is possible to define some recovery operators, like e.g. a consistency or a classicality operator $\circ A$-working such that $v(\circ A)=\mathbf{t}$ iff $v(A)=\mathbf{t}$ or $v(A)=\mathbf{f}$. It shall be noted that it is with the help of operators of this sort that advocates of Logics of Formal Inconsistency (LFIs, for short) recover what they have come to call gentle versions of classically valid inferences.

Thus, for example, though these systems invalidate Explosion-i.e. $A, \neg A \vDash B$ - they will still validate a restricted version of it, also known as Gentle Explosion-i.e. $\circ A, A, \neg A \vDash B .^{35}$ Along this line, it is with the help of a consistency operator that we could regain a restricted or gentle version of Modus Ponens - i.e. $\circ A, \circ B, A, A \rightarrow B \vDash B$-allowing us to operate prooftheoretically as intended. Incidentally, this observation shows a connection between the work we carried out in this paper, and the broader topic of recovery operators.

Finally, we could alternatively say that although the material conditional defined as $\neg A \vee B$ invalidates Modus Ponens in the functionally complete systems described above, there are plenty of definable conditionals which validate Modus Ponens - all of which can be used, perhaps more conveniently, for proof-theoretically and inferential purposes. As is explained by Hitoshi Omori in [25], central to the Goodship Project is not the fact that Modus Ponens is invalid tout court, but that it is invalid for the conditional employed in formulating the comprehension axioms of the naive theory in question - nothing precludes these theories from including plenty of other well-behaved conditionals, and no harm is caused by such an inclusion, as Omori shows. Exactly the same holds for our target functionally complete theories of truth, discussed in this paper.

\section{Conclusion}

In this paper we introduced the four-valued infectious logics $\mathbf{S}_{\mathrm{fde}}, \mathbf{d} \mathbf{S}_{\mathrm{fde}}, \mathbf{d} \mathbf{S}_{\mathrm{fde}}^{w}$, and $\mathbf{S}_{\mathrm{fde}}^{w}$ as the basis of the non-trivial transparent theories of truth $\mathbf{S}_{\text {fde }} \mathbf{T T}, \mathbf{d} \mathbf{S}_{\text {fde }} \mathbf{T T}, \mathbf{d} \mathbf{S}_{\text {fde }}^{w} \mathbf{T T}$ and $\mathbf{S}_{\text {fde }}^{w} \mathbf{T T}$. From a conceptual point of view, we inspired the four-valued approach as a means to treat different kinds of pathological sentences, i.e. paradoxical and hypodoxical sentences, in a distinctive way; namely, by allowing some of them to be truth-value gluts and some others to be truth-value gaps. Most importantly, we motivated the study of four-valued infectious logics as a means to implement the idea that the semantic pathology suffered by at least some pathological sentences is infectious, in the context of four-valued logics. This led us to consider logics where truth-value gaps are infectious, but gluts are not; logics where truth-value gluts are infectious, but gaps are not; and logics where both are infectious in some sense, although when these two values cross paths, one of them uniformly triumphs over the other. Even then, we did not try to defend or suggest to adopt any of these systems in particular. Our aim was to contribute with a number of formal tools to the literature, interesting and helpful for formalizing some intuitions regarding how pathological sentences behave.

Focusing now on the proof-theoretical side of the current investigation, we analyzed these logics and the respective transparent theories of truth built on top of them, and provided both limitative and positive results. Thus, on the one hand, we have shown that there are some severe limitations regarding the possibility of providing standard Gentzen-style sequent calculi for these theories - for which we properly dualized and extended the results given in [14]. On the other hand, we provided four-sided sequent systems for the target logics and theories of truth, arguing that from a philosophical point of view, these calculi enjoy some desirable formal properties such as (i) the subformula property, (ii) to introduce exactly one occurrence of a connective in their conclusion and (iii) not to mention any other connective anywhere else in their formulation.

\footnotetext{
${ }^{35}$ For more about LFIs, see Newton da Costa's seminal work on his $C$-systems in [24] and [23]. These systems were later developed in a systematic way by W. Carnielli, J. Marcos, M. Coniglio and others in e.g. [18], [17] and $[16]$.
} 


\section{Acknowledgments}

We would like to thank the members of the Buenos Aires Logic Group for helpful feedback concerning previous versions of this paper. Our thanks also go to three anonymous reviewers for their comments and suggestions to improve the paper. Finally, we would also like to thank the editors of the special issue, Eduardo Barrio and Walter Carnielli, for their assistance through the editorial process. While writing this paper, Bruno Da Ré and Damian Szmuc were enjoying a fellowship from the National Scientific and Technical Research Council of Argentina (CONICET).

\section{References}

[1] L. Åqvist. Reflections on the logic of nonsense. Theoria, 28(2):138-157, 1962.

[2] O. Arieli and A. Avron. The value of the four values. Artificial Intelligence, 102(1):97-141, 1998.

[3] F. G. Asenjo. A calculus for antinomies. Notre Dame Journal of Formal Logic, 16(1):103$105,1966$.

[4] F. G. Asenjo and J. Tamburino. Logic of antinomies. Notre Dame Journal of Formal Logic, 16(1):17-44, 1975.

[5] A. Avron. Natural 3-valued logics - characterization and proof theory. The Journal of Symbolic Logic, 56(01):276-294, 1991.

[6] A. Avron, J. Ben-Naim, and B. Konikowska. Cut-free ordinary sequent calculi for logics having generalized finite-valued semantics. Logica Universalis, 1(1):41-70, 2007.

[7] A. Avron and I. Lev. Canonical propositional gentzen-type systems. In International Joint Conference on Automated Reasoning, pages 529-544. Springer, 2001.

[8] E. Barrio, F. Pailos, and D. Szmuc. A Cartography of Logics of Formal Inconsistency and Truth. Unpublished Manuscript.

[9] E. Barrio, F. Pailos, and D. Szmuc. A paraconsistent route to semantic closure. Logic Journal of the IGPL, 25(4):387-407, 2017.

[10] J. Beall. Multiple-conclusion LP and default classicality. The Review of Symbolic Logic, 4(02):326-336, 2011.

[11] J. Beall, M. Glanzberg, and D. Ripley. Liar paradox. In E. N. Zalta, editor, The Stanford Encyclopedia of Philosophy. Stanford University, Winter 2016 edition, 2016.

[12] J. C. Beall. Spandrels of Truth. Oxford University Press, 2009.

[13] D. A. Bochvar. On a three-valued logical calculus and its application to the analysis of the paradoxes of the classical extended functional calculus. History and Philosophy of Logic, 2(1-2):87-112, 1981. Translated by M. Bergmann.

[14] S. Bonzio and M. Baldi. Undefinability of standard sequent calculi for paraconsistent threevalued logics. arXiv preprint arXiv:1612.00963, 2016.

[15] S. Bonzio, J. Gil-Férez, F. Paoli, and L. Peruzzi. On paraconsistent weak kleene logic: Axiomatisation and algebraic analysis. Studia Logica, 105(2):253-297, 2017.

[16] W. Carnielli and M. Coniglio. Paraconsistent Logic: Consistency, Contradiction and Negation. Springer International Publishing Switzerland, 2016.

[17] W. Carnielli, M. Coniglio, and J. Marcos. Logics of Formal Inconsistency. In D. Gabbay and F. Guenthner, editors, Handbook of Philosophical Logic, volume 14, pages 1-93. Springer, 2007.

[18] W. Carnielli and J. Marcos. A taxonomy of C-systems. In M. C. Walter Carnielli and I. D'Ottaviano, editors, Paraconsistency: The Logical Way to the Inconsistent, volume 2 
of Lecture Notes in Pure and Applied Mathematics, pages 1-94. Marcel Dekker, New York, NY, 2002.

[19] R. Ciuni. Conjunction in Paraconsistent Weak Kleene Logic. In P. Arazim and M. Dančák, editors, The Logica Yearbook 2014, pages 61-76. College Publications, London, 2015.

[20] R. Ciuni and M. Carrara. Characterizing Logical Consequence in Paraconsistent Weak Kleene. In L. Felline, A. Ledda, F. Paoli, and E. Rossanese, editors, New Directions in Logic and the Philosophy of Science, pages 165-176. College Publications, London, 2016.

[21] M. Coniglio and M. I. Corbalán. Sequent calculi for the classical fragment of Bochvar and Halldén's Nonsense Logics. In Proceedings Seventh Workshop on Logical and Semantic Frameworks, with Applications, LSFA 2012, Rio de Janeiro, Brazil, September 29-30, 2012., pages 125-136, 2012.

[22] M. E. Coniglio and L. H. D. C. Silvestrini. An alternative approach for quasi-truth. Logic Journal of IGPL, 22(2):387-410, 2014.

[23] N. da Costa. On the theory of inconsistent formal systems. Notre Dame Journal of Formal Logic, 15(4):497-510, 1974.

[24] N. da Costa. Sistemas formais inconsistentes (Inconsistent formal systems, in Portuguese). $\mathrm{PhD}$ thesis, Habilitation thesis, Universidade Federal do Paraná, Curitiba, Brazil, 1963. Republished by Editora UFPR, Curitiba, Brazil, 1993.

[25] M. De and H. Omori. Classical Negation and Expansions of Belnap-Dunn Logic. Studia Logica, pages 1-27, 2015.

[26] H. Deutsch. Paraconsistent analytic implication. Journal of Philosophical Logic, 13(1):1-11, 1984.

[27] M. Dummett. What is a Theory of Meaning? (II). In G. Evans and J. McDowell, editors, Truth and Meaning: Essays in Semantics, pages 67-137. Clarendon Press, Oxford, 1976.

[28] M. Dummett. The Logical Basis of Metaphysics. Duckworth, London, 1991.

[29] J. M. Dunn. Intuitive semantics for first-degree entailments and 'coupled trees'. Philosophical studies, 29(3):149-168, 1976.

[30] P. Eldridge-Smith. Paradoxes and hypodoxes of time travel. In J. L. Jones, P. Campbell, and P. Wylie, editors, Art and time, pages 172-189. Australian Scholarly Publishing, Melbourne, 2007.

[31] P. Eldridge-Smith. The liar paradox and its relatives. PhD thesis, The Australian National University, 2008.

[32] P. Eldridge-Smith. Two paradoxes of satisfaction. Mind, 124(493):85-119, 2015.

[33] T. M. Ferguson. A computational interpretation of conceptivism. Journal of Applied NonClassical Logics, 24(4):333-367, 2014.

[34] T. M. Ferguson. Faulty Belnap computers and subsystems of FDE. Journal of Logic and Computation, 2014.

[35] T. M. Ferguson. Logics of nonsense and Parry systems. Journal of Philosophical Logic, 44(1):65-80, 2015.

[36] T. M. Ferguson. Rivals to Belnap-Dunn Logic on Interlaced Trilattices. Studia Logica, 2016. Forthcoming.

[37] H. Field. Saving Truth from Paradox. Oxford University Press, New York, 2008.

[38] M. Fitting. Kleene's three valued logics and their children. Fundamenta informaticae, 20(1, 2, 3):113-131, 1994.

[39] L. Goodship. On dialethism. Australasian Journal of Philosophy, 74(1):153-161, 1996.

[40] A. Gupta and N. Belnap. The Revision Theory of Truth. MIT Press, Cambridge, Mass, 1993.

[41] A. Gupta and R. Martin. A Fixed Point Theorem for the Weak Kleene Valuation Scheme. Journal of Philosophical Logic, 13:131-135, 1984. 
[42] S. Halldén. The logic of nonsense. Uppsala Universitets Arsskrift, Uppsala, 1949.

[43] P. Horwich. Truth. Clarendon, Oxford, 1998.

[44] L. Humberstone. Power Matrices and Dunn-Belnap Semantics: Reflections on a Remark of Graham Priest. The Australasian Journal of Logic, 11(1):14-45, 2014.

[45] S. C. Kleene. Introduction to metamathematics. North-Holland, Amsterdam, 1952.

[46] S. Kripke. Outline of a theory of truth. Journal of Philosophy, 72(19):690-716, 1975.

[47] G. Malinowski. Many-valued logic. In D. Jacquette, editor, A Companion to Philosophical Logic, pages 545-561. Blackwell, 2002.

[48] R. L. Martin and P. W. Woodruff. On representing 'true-in-L' in L. Philosophia, 5(3):213217,1975 .

[49] J. McFarlane. Logical constants. In E. N. Zalta, editor, The Stanford Encyclopedia of Philosophy. Stanford University, Fall 2015 edition, 2015.

[50] H. Omori. Halldén's Logic of Nonsense and its expansions in view of Logics of Formal Inconsistency. In Proceedings of DEXA2016, pages 129-133. IEEE Computer Society, 2016.

[51] H. Omori and D. Szmuc. Conjunction and disjunction in infectious logics. In A. Baltag, J. Seligman, and T. Yamada, editors, Logic, Rationality, and Interaction: 6th International Workshop, LORI 2017, pages 268-283, Berlin, Heidelberg, 2017. Springer Berlin Heidelberg.

[52] F. Paoli. Tautological entailments and their rivals. In J.-Y. Béziau, W. A. Carnielli, and D. M. Gabbay, editors, Handbook of Paraconsistency, pages 153-175. College Publications, 2007.

[53] J. Peregrin. Inferentialism: Why Rules Matter. Palgrave Macmillan, 2014.

[54] E. Post. Introduction to a general theory of elementary propositions. Bulletin of the American Mathematical Society, 26:437, 1920.

[55] E. Post. Introduction to a general theory of elementary propositions. American Journal of Mathematics, 43(3):163-185, 1921.

[56] G. Priest. The logic of paradox. Journal of Philosophical logic, 8(1):219-241, 1979.

[57] G. Priest. Hyper-contradictions. Logique Et Analyse, 27(7):237-243, 1984.

[58] G. Priest. Paraconsistent logic. In D. Gabbay and F. Guenthner, editors, Handbook of Philosophical Logic, volume 6, pages 287-393. Springer, Dodrecht, 2002.

[59] G. Priest. In Contradiction: A Study of the Transconsistent. Oxford University Press, 2006.

[60] G. Priest. An Introduction to Non-Classical Logic: from If to Is. Cambridge University Press, Cambridge, 2nd edition, 2008.

[61] G. Priest. The logic of the catuskoti. Comparative Philosophy, 1(2):51-51, 2010.

[62] G. Priest. Plurivalent logics. The Australasian Journal of Logic, 11(1):2-13, 2014.

[63] G. Priest. None of the above: The catuskoti in indian buddhist logic. In J.-Y. Beziau, M. Chakraborty, and S. Dutta, editors, New Directions in Paraconsistent Logic, pages 517527. Springer India, New Delhi, 2015.

[64] G. Priest. What If? The Exploration of an Idea. The Australasian Journal of Logic, 14(1):54-127, 2017.

[65] W. V. Quine. Philosophy of Logic. Englewood Cliffs, NJ: Prentice-Hall, 1970.

[66] L. Rabern, B. Rabern, and M. Macauley. Dangerous reference graphs and semantic paradoxes. Journal of Philosophical Logic, 42(5):727-765, 2013.

[67] G. Restall. Multiple conclusions. In P. Hájek, L. Valdés-Villanueva, and D. Westerståhl, editors, Logic, Methodology and Philosophy of Science. College Publications, 2005.

[68] G. Restall. First degree entailment, symmetry and paradox. Logic and Logical Philosophy, 26:3-18, 2016.

[69] D. Ripley. Conservatively Extending Classical Logic with Transparent Truth. Review of Symbolic Logic, 5(2):354-378, 2012. 
[70] D. Ripley. Paradoxes and failures of cut. Australasian Journal of Philosophy, 91(1):139-164, 2013.

[71] R. Routley. Dialectical logic, semantics and metamathematics. Erkenntnis, 14(3):301-331, 1979.

[72] D. Szmuc. Defining LFIs and LFUs in extensions of infectious logics. Journal of Applied Non-Classical Logics, 26(4):286-314, 2017.

[73] N. Tennant. Existence and identity in free logic: A problem for inferentialism? Mind, 116:1055-1078, 2007.

[74] A. Visser. Four valued semantics and the liar. Journal of Philosophical Logic, 13(2):181-212, 1984.

[75] W. Wernick. Complete sets of logical functions. Transactions of the American Mathematical Society, 51:117-130, 1942.

[76] S. Wintein. On all strong kleene generalizations of classical logic. Studia Logica, 104(3):503$545,2016$. 